\title{
A conserved arginine in NS5 binds genomic 3'stem-loop RNA for primer- independent initiation of flavivirus RNA-replication
}

\section{Authors}

Sai Wang ${ }^{\mathrm{a}}$, Kitti Wing Ki Chan ${ }^{\mathrm{a}}$, Min Jie Alvin Tan ${ }^{\mathrm{a}}$, Charlotte Flory $^{\mathrm{a}}$, Dahai Luo ${ }^{\mathrm{c}}$, Julian Lescar ${ }^{\mathrm{d}}$, Jade K Forwood ${ }^{\mathrm{e}}$, Subhash G Vasudevan ${ }^{\star \mathrm{a}, \mathrm{fg}}$

a Program in Emerging Infectious Diseases, Duke-NUS Medical School, 8 College Road 169857, Singapore

c Lee Kong Chian School of Medicine, Nanyang Technological University, 59 Nanyang Drive, Singapore, 636921

'School of Biological Sciences, Nanyang Technological University, 60 Nanyang Drive, Singapore, 637551

e School of Biomedical Sciences, Charles Sturt University, Wagga Wagga, NSW 2650, Australia

${ }^{\mathrm{f}}$ Department of Microbiology and Immunology, National University of Singapore, 5 Science Drive 2, Singapore 117545

${ }^{9}$ Institute for Glycomics, Griffith University, Gold Coast Campus, QLD 4222, Australia

* Address correspondence to Subhash G. Vasudevan, subhash.vasudevan@dukenus.edu.sg

\section{Running title : Flaviviral de novo RNA replication}

\begin{abstract}
Replication of the RNA genome of flaviviruses without a primer involves RNA-protein interactions that have been shown to include the recognition of the stem-loop A (SLA) in the 5' untranslated region (UTR) by the non-structural protein 5 (NS5). We show that DENV2 NS5 arginine 888, located within the C-terminal 18 residues, is completely conserved in all flaviviruses and interacts specifically with the top-loop of 3'SL in the 3'UTR which contains the pentanucleotide 5'-CACAG-3' previously shown to be critical for flavivirus RNA replication. We present virological and biochemical data showing the importance of this Arg 888 in virus viability and de novo initiation of RNA polymerase activity in vitro. Based on our binding studies, we hypothesize that ternary complex formation of NS5 with 3'SL, followed by dimerization, leads to the formation of the de novo initiation complex that could be regulated by the reversible zipping and unzipping of cis-acting RNA elements.
\end{abstract}

Word count: 150 . 


\section{Introduction}

Dengue remains a major public health issue in more than 100 countries where twothirds of the world's population resides. The disease is caused by dengue virus (DENV) that is transmitted by Aedes mosquito. The four DENV serotypes (DENV 1-4) along with Zika virus (ZIKV), Yellow fever virus (YFV), West Nile virus (WNV), tickborne encephalitis virus (TBEV), and the zoonotic Usutu virus (USUV), are members of the flavivirus genus belonging to the family Flaviviridae ${ }^{1}$. Despite millions of infection cases each year, there are no specific antivirals against DENV or any flaviviruses. Hence there is an urgent need for therapeutic candidates against these viruses that is partly driven by vaccine rollout issues and other well-publicized limitations of approved vaccines such as Dengvaxia ${ }^{2}$.

The $11 \mathrm{k}$ nucleotide $(\mathrm{nt})$ positive-strand RNA genome of DENV contains a long open reading frame (ORF) that encodes a polyprotein that is post-translationally cleaved by viral and host proteases into three structural proteins (capsid, envelope and membrane) and seven non-structural proteins (NS1, NS2A, NS2B, NS3, NS4A, NS4B, and NS5). The ORF is flanked by 5' and 3' untranslated regions (UTR) that are $100 \mathrm{nt}$ and $450 \mathrm{nt}$ long respectively, which contain multiple secondary structure elements ${ }^{3,4}$. The two conserved stem-loop structures SLA and SLB in 5'UTR are required for viral RNA replication with SLA acting as a promoter that is believed to have a large binding footprint on NS5 ${ }^{5-8}$. SLB contains the sequence element known as 5' UAR (Upstream of ATG Region) that is complementary to a sequence within the 3'UTR involved in genome circularization, and is flanked by AU rich region that has been suggested to form a cis-acting conformationally tuned RNA element termed 5'UAR-flanking stem (UFS) ${ }^{9}$. The secondary structures in 3'UTR can be divided into three domains ${ }^{10}$. The first two domains contain stem-loop structures SLI and SLII and dumb-bell structures DB1 and DB2 ${ }^{11-14}$. The 3 'SL structure within Domain III of 3'UTR is highly conserved among flaviviruses and contains sequences that have been shown to interact with the DENV RNA-dependent RNA polymerase (RdRp) in a yeast 3-hybrid assay ${ }^{15}$ as well as being important for WNV replication but not translation ${ }^{16}$. In addition to the secondary structures, there are three pairs of complementary sequences (CS, UAR, DAR) from 5' and 3' regions of the genome which together with other functionally important long-range RNA-RNA interactions within the ORF region, drive genome circularization for initiation of replication ${ }^{7,8,17}$.

NS5 is the largest and most conserved DENV protein and is the key player in viral RNA replication. It is composed of two enzymatic domains, the $\mathrm{N}$-terminal methyltransferase (MTase) and C-terminal RNA-dependent RNA polymerase (RdRp), 18,19 and can also suppress host innate antiviral response ${ }^{20}$. The dynamic intramolecular interaction between the two NS5 domains is facilitated by the inter- 
domain linker region which is critical for DENV replication ${ }^{21-23}$. MTase domain catalyzes methylation of the viral genome 5' cap structure to form a type 1 cap, which ensures the stability and integrity of the genome ${ }^{24}$. RdRp adopts a canonical right-hand conformation consisting of three subdomains, thumb, fingers and palm, which carry out processive RNA synthesis. DENV RNA replication occurs within vesicle-like structures formed by the invagination of rough endoplasmic reticulum (ER) membrane within which the replication complex $(\mathrm{RC})$ consisting of viral nonstructural proteins as well as unknown host proteins orchestrate RNA replication ${ }^{25-28}$. Recently it was shown that the 3'SL is required for the formation of ER-derived vesicle packets in DENV infected cells ${ }^{29}$. Although NS5's role in RNA replication is carried out within these membrane restricted vesicles in the cytoplasm ${ }^{28}$, a large proportion of the protein is found in the nucleus of DENV2 infected cells ${ }^{30-33}$. The Cterminal $18\left(\mathrm{C}_{\text {ter }} 18\right)$ residues (882-900) of DENV2 NS5 contain a nuclear localization signal (NLS) sufficient for its recognition as a cargo by the host importin $\alpha$ nuclear transport protein for translocation to the nucleus ${ }^{34}$. Although the NLS function can be disrupted without affecting viral RNA synthesis by mutation of residues required for importin binding, mutation of R888, a residue completely conserved in all flaviviruses leads to non-viable DENV2 ${ }^{33}$. In addition, NS5 dimerization has been observed in proteins from both DENV and ZIKV and could be required for the formation of a cooperative complex of MTase and RdRp, enabling capping of newly synthesized RNA emerging from the RdRp in trans by the MTase domain from the other monomer ${ }^{35,36}$. The $\mathrm{C}_{\text {ter }} 18$ region is often not resolved in crystal structures of flaviviral NS5 ${ }^{22,37}$ except in the case where NS5 dimers were observed and the $\mathrm{C}_{\text {ter }} 18$ region contained an alpha helix that protruded from NS5 molecule into the MTase domain of the interacting NS5 partner ${ }^{35}$. Here R888 was found to be within $\mathrm{H}$-bonding distance to $\mathrm{Y} 838$ of the same monomer which is also completely conserved in all the major flavivirus phylogenetic groups (Supplementary Fig. 1) and has been identified as a potential RNA binding residue ${ }^{15}$.

It is widely accepted that recruitment of NS5 by 5'SLA acting as a promoter initiates negative strand RNA synthesis ${ }^{5-8,38}$. In contrast, how the SLA-recruited-NS5 translocates over to the 3' end to initiate primer-independent RNA synthesis on the 3'-end of the (-) RNA strand remains an enigma. Here, we used reverse genetics to show that R888 and the neighboring Y838 residue are critical for viable virus production and used RNA binding assays with NS5 and various NS5 mutants to identify a key structural interaction for the formation of a de novo RNA initiation complex between residue R888 at the NS5 C-terminal tail and the top-loop of 3'SL establish specific interactions. We propose a mechanistic model for flavivirus replication consistent with most recent structural and biological studies of NS5 and its RNA ligands. 


\section{Results}

\section{C-terminal Y838 and R888 play a critical role in DENV2 replication}

We previously identified the C-terminal 18 residues of DENV2 NS5 as a NLS recognised by host importins to transport the NS5 protein to the nucleus of infected cells. Arginine 888 forms the P3 residue of the NS5 NLS binding determinant for importin $\boldsymbol{\alpha}^{34}$. During evolution, this arginine residue has been strictly conserved in NS5 amino acid sequences from the four major flavivirus phylogenetic groups: mosquito-borne (MBFV), tick-borne (TBFV), insect-specific (ISFV) and no known vector (NKV) flaviviruses (Supplementary Fig. 1). Residue R888 interacts with residue Y838 (Figure 1A, Supplementary Fig. 1) in one of the molecules in the unit cell of DENV3 NS5 crystal structure where the C-terminal end is ordered via formation of a dimeric interaction ${ }^{35}$ while it is not observed in other structures because Cter18 is disordered ${ }^{22,37}$. To uncover any functional importance of this interaction, we introduced site-specific mutations into a DENV2 cDNA clone and examined their impact on virus replication and sub-cellular localization of NS5. Four single mutations Y838A, Y838F, R888A, or R888K were introduced individually into the DENV2 cDNA clone by site directed mutagenesis ${ }^{39}$. The infectious clone RNA containing each of these four mutations were generated by in vitro transcription (IVT) and transfected into BHK-21 cells to examine the replication kinetics over $96 \mathrm{~h}$. The replication deficient NS5 polymerase active site mutant "G662 DD" to "GAA" (referred to as GAA mutant) was included as negative control. BHK-21 cells transfected with Y838F IVT RNA showed $\sim 2-3$ fold lower viral RNA synthesis $(p<0.0001)$ and extracellular RNA ( $p=0.019$ ) level to that of wildtype (WT) (Figure 1B and C). However, the infectious virus was detected from $48 \mathrm{~h}$ post-transfection onwards with a $\sim$-fold lower infectious titer as compared to WT at $48 \mathrm{~h}(p=0.045)$ and $72 \mathrm{~h}$ posttransfection $(p=0.042)$ (Figure 1D), suggesting a lower infectivity of the resultant $\mathrm{Y} 838 \mathrm{~F}$ virus. This is supported by the observation of smaller plaque size (Figure 1E) and lower percentage infection of $\mathrm{Y} 838 \mathrm{~F}(30-40 \%)$ at $72 \mathrm{~h}$ post-transfection compared to WT (50-60\%) (Figure 2). On the other hand, R888K mutant exhibited severe attenuation in virus replication with $\sim 2$ log lower genome synthesis $(p<$ $0.0001)$, extracellular RNA ( $p=0.004)$ and infectious virus production $(p=0.023$ at $72 \mathrm{~h} ; p=0.017$ at $96 \mathrm{~h}$ post-transfection) compared to WT (Figure 1B-D). This slower replication kinetics of R888K is consistent with its smaller plaque phenotype compared to WT (Figure 1E) and low percentage infection (10-20\%) at $72 \mathrm{~h}$ posttransfection (Figure 2B). Similar to the replication defective GAA mutant, both Y838A and R888A mutants showed no increase in the viral RNA synthesis and extracellular RNA over $96 \mathrm{~h}$ (Figure 1B and C) that is consistent with lack of any plaque detection even without any dilution (Figure 1E), indicating that these mutations are lethal to the virus. Collectively these results suggest that mutations at these conserved positions of NS5 had an impact on the virus replication. 
We next examined the NS5 subcellular localization of its Y838F, R888K mutants in comparison to WT NS5 by confocal microscopy. In agreement with previous report ${ }^{34}$, NS5 of WT DENV2 was found mainly in the nucleus with a small fraction in the cytoplasm that co-localized with dsRNA to carry out genome replication (Figure 2A). Neither Y838F nor R888K mutations altered NS5 nuclear localization (Figure 2B and C). Interestingly although the NS5 mutants (R888K and Y838F) have lower percentage infectivity compared to WT, the NS5 fluorescence intensity of these mutants were comparable to WT (Figure 2D). Notably the R888K mutant exhibited a significantly lower double stranded (ds) RNA staining intensity compared to the WT $(p=0.03$; Figure 2E). This observation is consistent with the lower replication (Figure $1 \mathrm{~B}$ ) and infectivity (Figure $2 \mathrm{~B}$ ) of the R888K mutant, suggesting that the conserved arginine at position 888 may play a crucial role in viral RNA synthesis.

Overall, our data show that NS5 C-terminal residues Y838 and R888 play a critical role in DENV2 replication independent of R888's role as a determinant of NS5 subcellular localization ${ }^{34}$.

\section{NS5 Y838 and R888 play a critical role in RNA polymerase activities}

In order to explore a potential role of residues $Y 838$ and $R 888$ in viral RNA replication, the polymerase activity of the NS5 single mutants Y838F, R888A and R888K were examined by de novo initiation/elongation (referred to as dni/elongation) and elongation assays in vitro (Figure $3 \mathrm{~B}$ ) ${ }^{22,40}$. The recombinant DENV2 NS5 WT and mutant proteins generated by site-directed mutagenesis were expressed in E.coli (Figure 3A). The NS5 Y838A mutant was unstable and could not be purified which could account for this mutation being lethal in our transfection studies (Figure $1 \mathrm{~B}, \mathrm{C}$, and E). The enzymatic activity of each mutant NS5 protein was normalized against that of WT NS5 that were set as $100 \%$ (Figure 3B). Compared to WT, the dni/elongation polymerase activity of purified NS5 Y838F and R888K was reduced by about $40 \%$ which may be attributed to the breakdown of the hydrogen bond between the side chains of the two residues. On the other hand, the elongation activity of Y838F mutant protein was similar to WT, and that of R888K was slightly stimulated compared to WT. The NS5 R888A mutant protein resulted in a dramatic reduction in the dni/elongation polymerase activity by more than $80 \%$ compared to WT NS5, while the elongation activity was lowered by $30 \%$, suggesting that R888 may play a crucial role in de novo RNA initiation.

Since NS5 is a multifunctional protein where the $\mathrm{N}$ terminal MTase domain has been shown to modulate the C-terminal RdRp and vice versa ${ }^{21,41}$, we included the NS5 D146A (part of the MTase "KDKE" active active site ${ }^{40}$ ) MTase defective mutant protein, the $\mathrm{G}_{662} \mathrm{DD} \rightarrow \mathrm{GAA}$ RdRp defective mutant protein and a combined $\mathrm{D} 146 \mathrm{~A}+\mathrm{GAA}$ mutant in the polymerase activity assays. The MTase inactive D146A mutation led to significant decrease in both dni/elongation and primer-dependent elongation activities of NS5 polymerase by around $65 \%$ and $40 \%$, respectively, 
suggesting that MTase is in cross-talk with the RdRp activity through intramolecular signaling between the two domains which could potentially influence NS5's affinity for RNA substrate. The polymerase inactive GAA mutation completely abolished the dni/elongation activity as expected, however, it still retained around $10 \%$ of the elongation activity in vitro. The D146A+GAA mutant completely abolished both dni/elongation and elongation activities. Taken together the in vitro polymerase assays indicate that the NS5 residues Y838 and R888 play a role in RNA replication.

\section{C-terminal R888 modulates NS5 binding affinity with 5'SLA and 3'SL}

The precise role of $\mathrm{Y} 838$ and R888 of NS5 is not known but the dni/elongation polymerase activity assay results suggest that they play a role in RNA binding events associated with viral RNA replication. To examine this further, we investigated the interactions of the WT and mutant NS5 proteins with the conserved RNA secondary structural features in the 5'UTR and 3' UTR regions focusing on the 70 nt 5'SLA and 79 nt 3'SL respectively (Figure 4A). The secondary structures for the 5' SLA and $3^{\prime}$ 'SL were predicted using the mfold web server ${ }^{42}$, and the thermodynamic negative free energy of the folding process at $37^{\circ} \mathrm{C}$ suggests that the RNAs used in our study are folded into a stable structure.

5'SLA and 3'SL RNA were generated by in vitro transcription as described previously 43. The purity of RNA samples was analyzed by agarose gel electrophoresis as shown in Figure 4A. The RNA electrophoretic mobility shift assay (REMSA) was conducted to assess protein-RNA binding. Purified WT or mutant NS5 was mixed with a fixed amount of 5'SLA or 3'SL RNA at molar ratios ranging 0:1, 0.5:1, 1:1, 2:1, $4: 1,8: 1$ and $16: 1$, followed by electrophoresis in agarose gels and staining with GelRed for RNA visualization as shown in Figure 4B \& C. Samples of 0:1 molar ratio of NS5 and RNA mixture were loaded on lane 1 of each gel showing RNA alone in the binding buffer (50 mM HEPES pH 7.0, $150 \mathrm{mM} \mathrm{NaCl}, 10 \%$ Glycerol, $1 \mathrm{mM}$ TCEP and $0.5 \mathrm{mg} / \mathrm{ml} \mathrm{BSA}$ ). As the amount of NS5 was increased in the binding reaction, the intensity of the free RNA band at the bottom reduced, as the RNA binds to NS5 to form a complex that migrates slower. Taking the WT NS5 and 3'SL binding reactions as an example (Figure $4 \mathrm{C}$, left panel), the slow migrating NS5-RNA complex was faintly visible for the $0.5: 1$ ratio. At a 1:1 ratio, the free RNA intensity was reduced by around $40 \%$ and the larger band started appear as a broad smear. The intensity of the larger band was more prominent from 2:1 molar ratio of NS5 to RNA. To quantify the binding affinity, the size and band intensity of free RNA remaining for each reaction ratio was quantified with ImageJ software ${ }^{44}$. The RNA binding affinity was calculated for WT NS5 and each of the mutant with 5'SLA and 3'SL and presented as apparent dissociation constant $(\mathrm{Kd})$ in Figure 4D where tighter affinity corresponds to a smaller Kd value. The Kd value of WT NS5 with 5'SLA is in the micromolar range and is in a similar Kd range measured for ZIKV fulllength NS5 binding with ZIKV 5'SLA ${ }^{38}$. Mutation of residue R888 to either A or K resulted in a slightly higher binding affinity of NS5 with 5'SLA in comparison with WT, 
bearing in mind that REMSA is a semi-quantitative technique. Remarkably, NS5 R888A and R888K mutant proteins showed reduced binding affinity with 3'SL by over 3- and 2-fold, respectively compared to WT NS5. The NS5 Y838F mutation showed no significant change in binding with 5'SLA or 3'SL, again probably due to lack of sensitivity of REMSA. The binding of MTase and RdRp active sites mutants (D146A, GAA or D146A+GAA) with 5'SLA and 3'SL were similar to WT, suggesting that the defective enzymatic functions were not directly linked to NS5 binding interactions with 5'SLA and 3'SL. This may have implications for the recent finding that the 3' terminal sequence of DENV genome has a non-replicative role in the formation of membranous replications organelles ${ }^{29}$. When we incubated the WT and R888A NS5 proteins with yeast tRNA, we did not detect an appreciable interaction between yeast tRNA and both NS5 proteins (Supplementary Figure 2B). Furthermore, the addition of yeast tRNA at vast molar excess (> 100 fold) did not affect WT NS5's ability to bind 3'SL. Taken together, the binding we demonstrate between NS5 and 3'SL is specific and dependent on the R888 residue.

\section{5'SLA-bound NS5 complex has an affinity for the 3'SL-bound NS5}

In order to examine the interactions of 5'SLA and 3'SL in NS5 in solution using coimmunoprecipitation, we labelled 5'SLA with biotinylated cytidine by adding it to its 3' end with T4 RNA ligase. The biotinylated 5'SLA was immobilized onto the streptavidin Sepharose beads and incubated with NS5 at 2:1 molar ratio of NS5 to 5'SLA to from 5'SLA-NS5 complex. We found that removal of excess NS5 by stringent washing followed by addition of various amount (molar ratio of 3'SL to 5'SLA ranges from $0: 1$ to 4:1) of unlabeled 3'SL resulted in a significant decrease of SLA-bound NS5 at high 3'SL concentration ( $p<0.0001$ ) (Figure 5A). Interestingly, if 3'SL is added directly to the 5'SLA-NS5 mixture without the washing step to remove the excess NS5, we detect an increase in NS5 binding with increasing amounts of 3'SL (Figure 5B) ( $p<0.05$ ), suggesting additional association of NS5 to the 5'SLA attached beads facilitated by 3'SL most likely in the form of 3'SL-NS5 complex.

Taken together, the co-immunoprecipitation data suggests 3'SL can displace NS5 that is bound to immobilized 5'SLA when the free NS5 amount is limited. However in conditions where excess free NS5 is present together with 3'SL, a more intricate NS5-RNA complex forms that needs further investigation.

\section{C-terminal R888 is critical for the formation of NS5-3'SL complex}

After establishing that R888 may be crucial for dni/elongation of polymerase and also demonstrating that it dramatically decreased binding affinity for 3'SL, we carried out further co-immunoprecipitation studies to assess complex formation between the NS5 R888A mutant and 3'SL. A biotinylated cytidine was added to the 3' end of the 79 nt 3'SL RNA molecule with T4 RNA ligase in order to immobilize it on the streptavidin Sepharose beads. Binding of NS5 WT, R888A or GAA to the 
immobilized 3'SL were assessed after stringent wash steps to remove non-specific binding. The NS5 proteins bound to the beads were then analyzed by SDS-PAGE followed by Western blot detection using in-house NS5 5M1 antibody ${ }^{45}$ (Figure 6A). Direct binding of NS5 or mutants to mock coupled Sepharose beads (labeled as "noRNA") were carried out as control in parallel. The Western blot showed that WT NS5 was pulled-down by immobilized 3'SL specifically, suggesting that the RNA secondary structure was sufficiently exposed and available for NS5 binding in solution. The polymerase-inactive GAA mutant bound similarly implying that 3'SL binding occurred independently of the polymerase active site mutations consistent with the small increase in polymerase elongation assay in vitro (see above). In contrast, a greater than $70 \%$ reduction in pull-down was observed for R888A mutant (Figure 6B) showing that the arginine residue was a major determinant of NS5 recognition of the 3'SL. The presence of R888A mutant in the supernatant after centrifugation following the incubation with beads (Supplementary Fig. 3) demonstrated that the single alanine substitution did not impact NS5 stability or solubility.

To further evaluate the stable binding of NS5 to 3'SL in solution and compare it with the R888A mutant, dynamic light scattering (DLS) and gel filtration analysis of the protein-RNA complex were carried out. Figures 6C \& D show the intensity-weighted size distribution profiles of the particles present in samples of NS5 (WT or R888A), 3'SL and the mixture of NS5 and 3'SL with 1:1 molar ratio generated from the intensity profile of the scattered light at $25^{\circ} \mathrm{C}$. A $1: 1$ mixture of WT NS5 and 3'SL sample contained larger particles than WT NS5 or 3'SL alone (Figure 6C), suggesting the formation of WT NS5-3'SL complex. In contrast, the size distribution curve of the mixture of R888A with 3'SL (Figure 6D) almost overlapped with that of the R888A alone, indicating an absence in the formation of the R888A NS5-3'SL complex. Since the scattering intensity is determined by the 6th power of the size of

molecules ${ }^{46}$, the intensity-weighted size distribution curve of R888A ( 97\%) and 3'SL ( 3\%) mixture mainly represented the larger molecule R888A. To ensure a reliable estimation of particle size, WT NS5, R888A mutant and 3'SL RNA samples were measured twice at two different concentrations to eliminate concentration effect (Supplementary Fig. 4) ${ }^{46}$.

Taken together, our results from Co-IP and DLS suggested the R888A mutation abolished the formation of NS5-3'SL complex.

\section{NS5 R888 and the top-loop of 3'SL are the key players in NS5-3'SL interaction}

We next investigated whether the NS5-3'SL interaction is mediated by a specific sequence/structure within the 3'SL. The secondary structure of 3'SL (Figure 7A) as predicted by mfold $^{42}$ is composed of a long stem, a top loop and a side loop. The flavivirus-conserved sequence 5'-(C)ACAG-3' in the top-loop of 3'SL has been shown to be essential for WNV RNA synthesis ${ }^{16}$. In DENV, the 5'-ACAG-3' 
sequence has been suggested to interact with $\operatorname{RdRp}{ }^{15}$. Hence, we examined whether R888 in DENV2 NS5, which is conserved among four major groups of flaviviruses (MBFV, TBFV, ISFV, NKV; Supplementary Fig.1), is involved in the recognition of the top-loop in 3'SL. Two 3'SL deletion mutants were generated, to delete either the top-loop (3'SL-TL ${ }^{\text {del }}$ ) or the side-loop (3'SL-SL ${ }^{\text {del }}$ ) of 3'SL. The mfold 42 prediction for the loop-deleted RNA is shown in Figure 7A. RNA samples of 3'SL and its deletion mutants were produced by IVT as described previously ${ }^{43}$. The purity of RNA samples was analyzed by agarose gel electrophoresis as shown in Figure $7 \mathrm{~A}$.

The binding interactions of WT NS5 and R888A mutant with 3'SL, 3'SL-TL del, and 3'SL-SL ${ }^{\text {del }}$ were assessed by REMSA as previously described. Mixtures of NS5 and RNA with molar ratios ranging $0: 1,1: 1,2: 1,4: 1,8: 1$ and 16:1 were assessed by agarose gel electrophoresis followed by RNA visualization (Figure 7B \& C). WT NS5 binding to the 3'SL-SL ${ }^{\text {del }}$ was similar to that of 3'SL, as both 3'SL (lane 5) and 3'SL$S^{\text {del }}$ (lane 17) were almost depleted at a 4:1 molar ratio suggesting the formation of complex with WT NS5 (Figure 7C). In contrast, the majority of 3'SL-TL ${ }^{\text {del }}$ still remained as free RNA in lane 11. Correspondingly, the apparent dissociation constant Kd values (Figure 7D) suggested that deletion of side-loop from 3'SL had almost no effect on its binding affinity with WT NS5, while deletion of top-loop led to a dramatic reduction in the binding affinity with NS5 by over 3-fold. In contrast, neither the top-loop nor the side-loop had an impact on the binding of 3'SL with the R888A mutant as shown by the similar RNA band-shift patterns (Figure 7C) and the comparable Kd values (Figure 7D) among all three RNAs.

Taken together, NS5 residue R888 that forms part of the $\mathrm{C}_{\text {ter }} 18$ NLS is essential for the DENV2 virus life cycle due to its crucial role in de novo RNA synthesis modulated by the binding interaction with the top-loop region of 3'SL.

\section{Discussion}

We previously discovered that the C-terminal $18\left(\mathrm{C}_{\text {ter }} 18\right)$ residues of DENV NS5 protein played a crucial role in RNA replication, but the precise mechanism remained unclear at that time ${ }^{34}$. Within $\mathrm{C}_{\text {ter }} 18$, amino acids $886-M K R F R-890$ of DENV2 NS5 were determined by crystallography to be the binding region (P1-P5) to host importin $\boldsymbol{\alpha}$ nuclear transport protein ${ }^{34}$. Introduction of the R888A mutation into a DENV2 infectious clone was lethal, while the R888K mutant was severely attenuated even though it was still localized in the nucleus (Figure 2). We therefore hypothesized that the DENV2 NS5 residue R888, which is completely conserved among the four major flaviviral phylogenetic groups (MBFV, TBFV, ISFV, NKV), plays a crucial role in RNA 
replication (Supplementary Figure 1B). The C-terminal region of NS5 is often not observed in NS5 crystal structures as it is considered to be a highly dynamic part of the protein located within the thumb subdomain ${ }^{22}$. The high mobility/dynamics of the thumb subdomain was also shown previously by hydrogen-deuterium exchange mass spectrometry and consistent with the higher $\mathrm{B}$ factor of the region in the crystal structure ${ }^{22}$. We found that the alanine mutation of $Y 838$ was lethal to the virus although the Y838F was only slightly attenuated in agreement with its potential RNA binding role. Unexpectedly the NS5 Y838A recombinant protein could not be expressed in E.coli while the Y838F mutant protein was readily purified for biochemical studies. It is possible that the interaction of $Y 838$ with a structured region of the viral genomic RNA may assist in its folding, although this needs to be experimentally evaluated in the future. However purified NS5 proteins carrying the mutations at R888A and Y838F were significantly affected (R888A>Y838F) in de novo initiation/elongation polymerase activity compared with primer-dependent polymerase elongation activity (Figure 3 ). These virological and in vitro biochemical assays pointed to an important mechanistic role for R888 in binding to a specific RNA structural element during replication. The REMSA binding studies showed that the R888A mutation led to a very significant loss of binding to the 3'SL element in comparison to the Y838F, D146A (MTase deficient mutant), GAA (RdRp deficient mutant) or the D146 + GAA mutations. In an in vitro biochemical setting, the R888 binding to 3'SL is not dependent on the polymerase active site since the RdRp deficient NS5 GAA mutant and WT are pulled-down similarly by 3'SL immobilized on beads, while R888A mutant protein pull-down is very much diminished. Taken together, the dramatic decrease in the R888A mutant's binding affinity with 3'SL demonstrated by REMSA, RNA immunoprecipitation, and DLS analysis, and its $>80 \%$ decrease in de novo initiation/elongation polymerase activity suggest a critical role of R888 in viral replication through specific interactions with 3'SL. Furthermore, deletion of the top-loop from the 3'SL structure predicted by MFold ${ }^{42}$ resulted in a drastic decrease in NS5 binding affinity with 3'SL, in agreement with the finding that the deletion of pentanucleotide 5'-CACAG-3' in the analogous region of the 3'UTR region of WNV was essential for RNA replication but not required for translation ${ }^{16}$. From these studies, we can posit that the pentanucleotide within the top loop is recognized by NS5 through specific protein-RNA interactions.

So, what is the crucial function of R888 in flaviviral RNA replication? The flaviviral genome contains highly structured UTRs and complementary sequences at 5' and 3' ends that promote genome circularization for replication ${ }^{5-7,10,47,48}$ and numerous dynamic long-range RNA interactions $>4 \mathrm{k}$ bases apart within the ORF of the genome that appear to be functionally important ${ }^{49}$. The 5'SLA is the promoter and key element for flaviviral genome replication ${ }^{5-8}$. The NS5 binding to 5'SLA together with the genome cyclization sequences juxtaposes the 3' end of the viral RNA genome adjacent to the SLA-bound NS5 for NS5's translocation to the 3' end for de novo 
primer-independent RNA synthesis. However there is a gap in our knowledge about the atomic details of how NS5 specifically binds to SLA and/or how it translocates to the 3' end to initiate de novo RNA replication. Studies with ZIKV NS5 suggest that both the thumb subdomain and the MTase region are involved in binding SLA ${ }^{38}$ which potentially could permit the translocation of SLA-bound NS5 to the 3' end for de novo initiation and transcription of the new RNA strand. Interestingly the 5' promoter element binding to NS5 has been further refined by the finding that the 5'UTR includes a conformationally-tuned cis-acting AU-rich RNA element called 5' UAR flanking stem (UFS) that is critical for NS5 recruitment and replication ${ }^{9}$. The model proposed by Liu and colleagues ${ }^{9}$ does not consider how a 5' SLA-bound NS5 can translocate to the 3' end for de novo initiation and RNA genome replication. While other RNA viruses have evolved specific mechanisms for this crucial step ${ }^{50}$, there is no evidence for sequence-specific RNA interactions to account for flaviviral de novo initiation in the published literature.

In hepatitis $\mathrm{C}$ virus (HCV), a Flaviviridae family member, the $\mathrm{C}$ terminus of the $\mathrm{RdRp}$ and the $\boldsymbol{\beta}$-loop are thought to occlude the active site and the entry of RNA template as well as incoming and priming nucleotides. The dynamics of the $\mathrm{C}$ terminus and gate keeping by the $\boldsymbol{\beta}$-loop enable formation of a de novo initiation complex that undergoes conformational transition to attain a processive elongation state ${ }^{50}$. Based on our demonstration of sequence-specific RNA-binding of NS5 R888 to 3'SL (Figure 8), we propose the following scenario. 1) Once the viral genomic RNA extends and serves as a template for translation by host machinery, sufficient polyproteins are synthesized that are post-translationally cleaved by viral and host proteases. The resulting NS5 proteins bind at the 5' UTR via SLA ${ }^{5-7}$ stabilized by UFS (which also includes SLB) (Supplementary Fig. 5), and at the 3'UTR via the top loop of 3'SL (this study). Translation will be blocked by 5' UTR-NS5 interaction (this includes SLA and SLB) ${ }^{9}$ thus permitting the genome circularization via extensive long range RNA interactions ${ }^{49}$, the $5^{\prime}$ and 3 ' cyclization and DAR sequences ${ }^{47} .2$ ) Next the NS5 molecules bound to the 5' and 3' RNA structures associate into a larger complex as supported by our observation that NS5 loaded on to sepharose bead with immobilized 5' SLA binds more of the excess NS5 when free 3'SL is added in a dose-dependent manner (Figure 5). As mentioned, the DENV NS5 dimerization was first observed in the crystal structure obtained by Choi and colleagues ${ }^{35}$ which supports our observation of increased NS5 binding in our RNA pull down experiment (Figure 7B). 3) As a multi-domain protein where both the MTase and RdRp domains of NS5 are in constant intramolecular communication $21,38,41$, we posit that the two NS5 molecules will undergo conformational stabilization that positions the template 3' end and incoming nucleotides within proximity of the active site. 4) Distinct from the proposal by Liu and colleagues ${ }^{9}$, we hypothesize that the zipping and unzipping of the AU rich UFS may provide the crucial thermodynamic energy for the formation of the stable de novo initiation complex. Liu 
et al ${ }^{9}$ elegantly showed through reverse genetics that both disrupting and stabilizing the base-pairing of UFS which are completely conserved in all flaviviruses were lethal. The AU rich sequence is therefore most probably energetically favoured to zip and unzip to permit the 3'SL bound NS5 to add the incoming nucleotide to the dinucleotide primer ${ }^{51}$ in the de novo initiation process that could occur through defined steps that remain unknown without the necessary RNA-protein structures. 5) Once the de novo initiation steps are completed, the 3'SL bound NS5 interacts with NS3 ${ }^{39,52-54}$ and enters processive RNA replication.

Overall, we demonstrate in this work for the first time that DENV2 NS5 residue R888 which is conserved in all flaviviruses specifically recognises the 3'SL top-loop, which is essential for de novo initiation of genome replication. We also propose a highly plausible model depicting the formation of a stabilized dni complex mediated by both 5'SLA and 3'SL RNA that requires further biophysical validation that are beyond the scope of the current study. Given the highly conserved property of both R888 and 3'SL, our findings also pave the way towards development of a pan-flavivirus therapeutic solution targeting the critical de novo initiation step in RNA replication.

\section{Methods}

\section{Cells}

BHK-21 cells (baby hamster kidney fibroblast cells; ATCC) were maintained in RPMI 1640 medium (Gibco) supplemented with $10 \%$ [v/v] fetal bovine serum (FBS) and 1\% $[\mathrm{v} / \mathrm{v}]$ penicillin-streptomycin $(\mathrm{P} / \mathrm{S})$ at $37^{\circ} \mathrm{C}$ with $5 \% \mathrm{CO}_{2}$.

\section{DENV infectious clone generation}

Full-length DENV2-3295 infectious cDNA clone (GenBank accession: EU081177) used in this study has been previously described ${ }^{39}$. pWSK29 D2 fragment $3^{34,39}$ was used to introduce the respective NS5 mutations (Y838F, Y838A, R888K, R888A and G662DD $\rightarrow$ GAA) by QuikChange II XL site-directed mutagenesis kit (Agilent Technologies) following product manual. Primer sequences used here are available upon request. Sequences of the resulting mutants were confirmed by DNA sequencing service provided by 1st BASE. Fragment 3 containing the desired mutations was excised from the vector by Xbal and Sacl and cloned into pWSK29 D2 fragment $1+2$ plasmid that was similarly cut with Xbal and Sacl.

\section{Transfection and in vitro virus replication assay}

The full-length cDNA clones (WT and NS5 mutants) were linearized by Sacl and purified using phenol-chloroform method. RNA was then in vitro transcribed from the linearized plasmid using T7 mMESSAGE mMACHINE kit (Ambion). The in vitro transcribed RNA was transfected into BHK-21 cells using the previously described electroporation conditions to examine the replication profile of the viruses over $96 \mathrm{~h}$ 39. Supernatants were collected at indicated time-points for plaque quantification by standard BHK-21 plaque assay and extracellular viral RNA by real-time RT-PCR. 
Cells were then washed once with PBS prior to lysing with Trizol for quantification of intracellular viral RNA by real-time RT-PCR.

\section{RNA extraction and Real-time RT-qPCR}

For extracellular viral gRNA quantification, viral RNA from the supernatants were extracted by QIAamp Viral RNA extraction kit (Qiagen) according to the manufacturer's instructions. Viral load in the supernatants was obtained by real-time RT-qPCR in Bio-Rad real-time thermal cycler CFX96 using iTaq Universal SYBR green one step kit (Bio-Rad) using primers 5'-CAGGCTATGGCACTGTCACGAT-3' and 5'- CCATTTGCAGCAACACCATCTC-3' targeting the DENV2 envelope protein region ${ }^{43}$. Absolute viral RNA genome copy was calculated based on the standard curve generated from in vitro RNA product of DENV2-3295 E gene and reported as genome copy per $\mathrm{mL}$ of supernatant ${ }^{39,55}$.

Total RNA was isolated from cell lysates using TriZol (Invitrogen) extraction method and subjected to cDNA synthesis using ImProm-II Reverse Transcription System (Promega) according to manufacturer's instructions. Quantification of intracellular viral gRNA was conducted by real-time PCR using iQ SYBR Green Supermix (BioRad). PCR product of DENV2-3295 E gene was used to generate the standard curve for quantification of viral genome copy. The values of intracellular viral RNA genome copies were normalized to actin expression and reported as genome copy per $\mu \mathrm{g}$ of total RNA.

\section{Plaque assay}

$2 \times 10^{5}$ BHK-21 cells were seeded into a 24-well plate and incubated overnight at $37^{\circ} \mathrm{C}$ in $5 \% \mathrm{CO}_{2}$. Viral supernatants were serially diluted with serum free RPMI 1640 media and inoculated onto the cell monolayer for $1 \mathrm{~h}$. Virus inoculums were then removed, and cells were overlaid with $0.8 \%$ carboxylmethyl cellulose (CMC) and further incubated for 4-5 days. Infected cells were fixed with 3.7\% formaldehyde and stained with $1 \%$ crystal violet for plaque visualization.

\section{Immunofluorescence assay}

Transfected cells on coverslips were fixed with ice-cold methanol at $-20^{\circ} \mathrm{C}$ for $15 \mathrm{~min}$. Antibodies against DENV NS5 (human monoclonal IgG clone, 5R3 ${ }^{45}$ and dsRNA (Scicons) were used for detection of the viral antigens. Digitized images were captured using Zeiss LSM710 upright confocal microscope (Carl Zeiss, Germany) at $64 \times$ magnification. Image processing and fluorescence intensity quantification was done with ImageJ software ${ }^{56}$.

\section{RNA production}

5'SLA and 3'SL RNA

The 70 nt-long 5'SLA 
(5'-

AGTTGTTAGTCTACGTGGACCGACAAAGACAGATTCTTTGAGGAAGCTAAGCTT AACGTAGTTCTAACAG-3')

and 79 nt-long 3'SL

(5'-

AGATCCTGCTGTCTCCTCAGCATCATTCCAGGCACAGAACGCCAGAAAATGGAA TGGTGCTGTTGAATCAACAGGTTCT-3') RNAs were generated by in vitro transcription using MEGAscript Kit (Life technologies) following the product manual. The DNA templates were PCR amplified from a mini-replicon construct followed by purification by gel extraction using QIAquick Gel Extraction Kit. The synthesized RNAs were purified by lithium chloride precipitation method following the MEGAscript Kit (Life technologies) manual followed by analysis on a $1.5 \%$ agarose gel with 1x TAE buffer under 100V for 1h. GelRed (Biotium) was added into agarose at $1: 10000 \mathrm{v} / \mathrm{v}$ right before gel casting for detection of RNA using Chemidoc Imaging system.

DENV2 mini-replicon RNA as template for polymerase de novo initiation/elongation assay

A mini-replicon RNA containing 5' and 3' UTR and part of the capsid coding sequence (Supplementary Fig. 5A) was used as template for the in vitro polymerase de novo initiation/elongation assay. The mini-replicon RNA was synthesized and capped with $\mathrm{m}^{7} \mathrm{G}\left(5^{\prime}\right) \mathrm{ppp}\left(5^{\prime}\right) \mathrm{A}$ cap structure analog (New England Biolabs) by in vitro transcription using the MEGAscript Kit (Life technologies) following the product manual. RNA purification and agarose gel electrophoresis were carried out as described above.

3'SL RNA with top-loop (3'SL-TL ${ }^{\text {del }}$ ) or side-loop (3'SL-SL ${ }^{\text {del }}$ ) deletion

Deletion of sequences for top-loop (7nt 5'-ACAGAAC-3') or side-loop (6nt 5'AGAAAA-3') from a template DNA construct which is part of the 3'UTR was done by overlap PCR. After successful construction of 3'SL-TL del and 3'SL-SL del DNA templates, RNA synthesis and purification were done as mentioned above.

\section{RNA biotinylation}

Biotinylation of the purified 5'SLA and 3'SL RNA was carried out using the Thermo Scientific Pierce RNA 3' End Biotinylation Kit. A single biotinylated cytidine (bis)phosphate nucleotide was ligated to the 3' terminus of the single RNA strand by T4 RNA ligase followed by purification steps to remove enzyme and other components remained in the ligation reaction according to the product manual.

\section{Prediction of RNA secondary structure}

The secondary structures for the 5' SLA and 3'SL were predicted with mfold web server ${ }^{42}$ and the thermodynamic free energy of the folding process at $37{ }^{\circ} \mathrm{C}$ was 
calculated based on the nearest-neighbour energetics and further refined by efn2 program based on coaxial stacking ${ }^{57}$.

\section{Site-directed mutagenesis}

The Y838A, Y838F, R888A, R888K, D146A, G662DD $\rightarrow$ GAA and D146A+GAA mutations on DENV2 NS5 were done using QuikChange II XL site-directed mutagenesis kit (Agilent Technologies) following product manual. Primer sequences used here are available upon request. Sequences of the resulting mutants were confirmed by DNA sequencing service provided by 1st BASE.

\section{Expression and purification of DENV2 recombinant WT NS5 and mutants}

The NS5 coding sequence was constructed in pProEX-HTb vector with a N-terminal His6 tag followed by TEV cleavage site. Expression and purification of the recombinant full-length NS5 of DENV2 has been described previously ${ }^{22,58}$. E.coli cells were resuspended in His Buffer A (50 mM Tris- $\mathrm{HCl}$ pH 7.5, $500 \mathrm{mM} \mathrm{NaCl}, 10 \%$ glycerol, $10 \mathrm{mM} \beta$-mercaptoethanol, $10 \mathrm{mM}$ imidazole) and lysed with 0.1 volume of 10x FastBreak Cell Lysis Reagent (Promega), followed by DNase treatment and clarified by centrifugation. The supernatant was filtered by a $0.45 \mu \mathrm{m}$ syringe filter unit and loaded onto a HisTrapFF column and eluted by a linear gradient of imidazole from 10 to $500 \mathrm{mM}$. Fractions containing His-tagged protein were bagged into a SnakeSkin (Thermo Scientific, $30 \mathrm{k} \mathrm{MWCO}$ ) membrane tubing together with TEV for His tag cleavage and concomitantly dialyzed against His buffer $A$ at $4^{\circ} \mathrm{C}$ for $18 \mathrm{~h}$ or overnight. The cleavage mixture was re-loaded onto the HisTrapFF column to separate any possible un-cleaved NS5 protein. The flow-through sample from second run of His affinity chromatography was concentrated and loaded onto a HiPrep 26/60 Sephacryl S-200 HR column (GE Healthcare Lifesciences) which was equilibrated in S200 Buffer (20 mM HEPES pH 7.5, $300 \mathrm{mM} \mathrm{NaCl}, 10 \%$ glycerol, 2 $\mathrm{mM}$ DTT) for further purification by size exclusion chromatography. The purity of elution fractions was confirmed by SDS-PAGE.

\section{NS5 in vitro activity assay}

Both the de novo initiation/elongation assay (also called primer-independent polymerase assay) and the elongation assay (also called primer-dependent polymerase assay) have been described previously ${ }^{22,34}$. Briefly, the de novo initiation/elongation assay reaction consists of 100 nM DENV2 NS5, $100 \mathrm{nM}$ minireplicon RNA (with sequence and features shown in Supplementary Fig. 5A), $20 \mu \mathrm{M}$ ATP, $20 \mu \mathrm{M}$ GTP, $20 \mu \mathrm{M}$ UTP, $5 \mu \mathrm{M}$ BBT-CTP (Jena Bioscience), in a buffer containing $50 \mathrm{mM}$ Tris- $\mathrm{HCl}, \mathrm{pH} 7.5,10 \mathrm{mM} \mathrm{KCl}, 1 \mathrm{mM} \mathrm{MgCl}_{2}, 0.3 \mathrm{mM} \mathrm{MnCl}_{2}, 0.001 \%$ Triton $\mathrm{X}-100$ and $10 \mu \mathrm{M}$ cysteine. The elongation assay reaction consists of $100 \mathrm{nM}$ DENV2 NS5, $100 \mathrm{nM}$ polyU RNA template as shown in Supplementary Fig. 5B, 3 $\mu \mathrm{M}$ BBT-ATP (Jena Bioscience), in a buffer containing $50 \mathrm{mM}$ Tris- $\mathrm{HCl}, \mathrm{pH} 7.5,10$ $\mathrm{mM} \mathrm{KCl}, 0.5 \mathrm{mM} \mathrm{MnCl} 2,0.001 \%$ Triton X-100 and $10 \mu \mathrm{M}$ cysteine. 
Both assay reactions were incubated at $37^{\circ} \mathrm{C}$ for exactly $1 \mathrm{~h}$, terminated by addition of $10 \mu \mathrm{l}$ 2.5x STOP buffer (200 mM NaCl, $25 \mathrm{mM} \mathrm{MgCl}$, $1.5 \mathrm{M} \mathrm{DEA}, \mathrm{pH}$ 10) supplemented with $25 \mathrm{nM}$ calf intestinal alkaline phosphatase (CIP, New England Biolabs) to remove the pyrophosphate (PPi) from the BBT group. The amount of fluorescent BBT group was measured from a 384-well flat-bottom, black plate (Corning) by Tecan Spark $10 \mathrm{M}$ microplate reader at excitation and emission wavelengths of $422 \mathrm{~nm}$ and $566 \mathrm{~nm}$, respectively. Each tested condition was recorded from triplicate reactions.

\section{RNA-protein complex formation and RNA electrophoretic mobility shift assay (REMSA)}

The protocol of REMSA has been described previously ${ }^{58}$. Briefly, a set of RNA-NS5 binding interactions were set up in binding buffer $(150 \mathrm{mM} \mathrm{NaCl}, 1 \mathrm{mM}$ TCEP, 10\% Glycerol, $0.5 \mathrm{mg} / \mathrm{mL}$ BSA, $50 \mathrm{mM}$ HEPES $\mathrm{pH} 7.0$ ) with the molar ratio of NS5 to RNA ranges from $0: 1$ to $16: 1$ in total of $25 \mu \mathrm{L}$, in which the RNA was fixed at $160 \mathrm{nM}$ and NS5 varied from 0 to $2.56 \mu \mathrm{M}$. The binding reactions were seated on ice for 20 min and loaded to agarose gel run under $100 \mathrm{~V}$ for $100 \mathrm{~min}$. GelRed (Biotium) was added into the agarose at 1:10000 v/v right before gel casting for RNA and RNAprotein complex detection using Chemidoc Imaging system (Bio-Rad) with adjusted exposure time to avoid oversaturation of the RNA bands. Formation of RNA-protein complex was identified based on the retardation of RNA on the native agarose gel. Band intensity of the unbound free RNA was quantified by ImageJ. Amount of NS5bound RNA was calculated by subtracting the amount of unbound RNA from the total RNA input. Percentage of NS5-bound RNA was plotted against NS5 concentration, from which the apparent $K_{d}$ value was calculated by GraphPad Prism 5 using the equation $Y=B_{\max }{ }^{*} X^{h} /\left(K_{d}{ }^{h}+X^{h}\right)$, where $Y$ is the percentage of NS5-bound RNA, Bmax is the maximum specific binding in the same unit as $Y, X$ is the concentration of NS5 protein, $\mathrm{h}$ is the Hill slope defining the cooperativity of the binding interaction, $\mathrm{K}_{\mathrm{d}}$ is the protein concentration required to achieve half-maximum binding at equilibrium, expressed in the same unit as $X$.

\section{RNA-NS5 Co-immunoprecipitation (Co-IP)}

25 pmole of biotinylated RNA was added to $100 \mu \mathrm{L}$ of streptavidin beads (GE Healthcare Streptavidin HP) pre-equilibrated in buffer $0(150 \mathrm{mM} \mathrm{NaCl}, 50 \mathrm{mM}$ Tris, $\mathrm{pH}$ 7.5) and incubated at $4{ }^{\circ} \mathrm{C}$ for $30 \mathrm{~min}$ with gentle rotation followed by blocking with $2 \mathrm{mM}$ biotin in buffer 0.25 pmole of WT NS5 or mutant was added to the RNA tagged beads which were re-equilibrated with buffer 1 (150mM NaCl, $1 \mathrm{mM}$ TCEP, $0.5 \mathrm{mg} / \mathrm{mL}$ BSA, $1 \%$ Triton X-100, 50mM Tris, $\mathrm{pH} 7.5$ ) and incubated for $1 \mathrm{~h}$ with gentle rotation at $4{ }^{\circ} \mathrm{C}$. After being washed with buffer 1 and precipitated by centrifugation at $150 \mathrm{~g}$ for $1 \mathrm{~min}$, the beads were boiled in SDS loading buffer and analyzed by SDS-PAGE and Western blot detection using the in-house NS5 antibody $5 \mathrm{M} 1{ }^{45}$. 


\section{Dynamic light scattering (DLS)}

DLS was used to generate the intensity-weighted size distribution of WT NS5, NS5 R888A mutant, 3'SL RNA, and 1:1 molar ratio mixture of WT NS5 or R888A with 3'SL. $15 \mu \mathrm{L}$ of sample in DLS buffer (100 mM NaCl, $1 \mathrm{mM}$ TCEP, $50 \mathrm{mM}$ HEPES, $\mathrm{pH}$ 7.0) was loaded into a fluorescence quartz cuvette (Hellma) and measured by Zetasizer NanoS (Malvern) equipped with a backscatter detection system at 173 degree at $25^{\circ} \mathrm{C}$. WT NS5 at 1.3 and $0.65 \mathrm{mg} / \mathrm{mL}$, R888A mutant at 1.94 and 0.65 $\mathrm{mg} / \mathrm{mL}$, 3'SL RNA at 1.45 and $0.72 \mathrm{mg} / \mathrm{mL}$ were measured multiple times independently to eliminate concentration effect on the estimation of particle size. The hydrodynamic radius $R_{H}$ of the particle which is defined as the radius of a sphere having the same diffusion rate as the particle was calculated by Stokes-Einstein equation $R_{H}=k_{B} T / 6 \pi \eta D$, where $k_{B}$ is the Boltzmann coefficient, $T$ is the absolute temperature, $\eta$ is solvent viscosity, $D$ is diffusion coefficient which is determined from the auto-correlation function describing the fluctuation of scattered light. The scattering profile was analyzed by Zetasizer Nano software (version 6.01) using the non-negative least-squares algorithm to deconvolute the correlation curve to intensity-weighted size distribution.

\section{Statistical analysis}

The statistical significance of difference between two groups was evaluated using student $t$-test with two-tailed distribution and two-sample method assuming unequal variances. Two-way ANOVA with Bonferroni correction was performed to determine significant differences between groups for the intracellular and extracellular viral RNA kinetics data. $P$-values $<=0.0001,<=0.001,<=0.01,<=0.05$ were represented by ${ }^{* * * *},{ }^{* * *},{ }^{* *},{ }^{*}$, respectively; $P$-values $>0.05$ were not considered statistically significant.

\section{Figure Legends}

Figure 1. Mutational analysis of NS5 C-terminal residues Y838 and R888 in DENV2 by reverse genetics [A] 3D structure of a DENV3 NS5 monomer (PDB access code: 5 CCV.A ${ }^{35}$ ) with an enlarged view of the NS5 C-terminal region showing hydrogen bond formation between Tyrosine 838 and Arginine 888 through their side chains. Replication characteristics of DENV2 WT NS5 and NS5 mutants shown in [B]- [E]. BHK-21 cells were electroporated with $10 \mu \mathrm{g}$ of DENV2 WT or mutant infectious clone RNA and the replication kinetics was followed until $96 \mathrm{~h}$ posttransfection. The replication deficient NS5 mutant GAA was included as negative control. [B] Real-time PCR quantification of intracellular viral RNA at the indicated timepoints. The dotted line represented the detection level for mock-transfected cells. [C] Real-time PCR quantification of extracellular viral RNA in the supernatants of the transfected cells at the indicated timepoints. The dotted line represented the detection level in the supernatant of mock-transfected cells. [D] Virus titer in the supernatants of transfected BHK-21 cells measured by plaque assay. [E] Pictures showing plaque morphologies of DENV2 WT and NS5 mutant viruses. The dilution at which plaques can be observed are indicated. The data in [B-D] are presented as average \pm SD from two independent experiments. Differences in intracellular $[B]$ and 
extracellular [C] viral RNA kinetics between groups were compared by two-way ANOVA with Bonferroni correction. Mean values of the virus titers [D] between WT and the respective mutants are compared by unpaired Student t-test, and a $P$ value $<0.05$ was considered significant $(*, P<0.05 ; * *, P<0.01 ; * * *, P<0.0001)$.

Figure 2. Subcellular distribution of NS5 and dsRNA in WT and mutant DENV transfected cells. BHK-21 cells were transfected with DENV2 WT [A], R888K [B], and $\mathrm{Y} 838 \mathrm{~F}$ [C] mutants as in Fig. 1B and analyzed at $72 \mathrm{~h}$ post transfection for NS5 (green) and dsRNA (red) by immunofluorescence assay. Digitized images were captured by Zeiss LSM710 upright confocal microscope using a $63 \times$ oil immersion lens. The \% infectivity are indicated, and the insets in the bottom panes show the zoom-in views of the dotted box regions. Two independent experiments were performed and the representative images were observed for more than $99 \%$ of the cells. Intensity quantification of [D] NS5 and [E] dsRNA fluorescence of WT, R888K and Y838F infected cells. Data is presented as bar graphs with average $\pm S D$ obtained from $n=30$ infected cells ( 9 field images for WT, 24 field images for R888K and 15 filed images for Y838F). Values indicated on top of the bars of graph in [D] are the \% infectivity of the respective viruses. Difference in fluorescence intensity between WT and R888K or Y838F were compared by Student t-test and a $P$ value < 0.05 was considered significant $(* P<0.05)$.

Figure 3. Assessment of primer-independent de novo initiation/elongation and primerdependent elongation polymerase activities of WT NS5 and mutant. [A] SDS-PAGE analysis of WT NS5 and NS5 mutant recombinant proteins expressed in E.coli and purified by His-tag affinity and size exclusion chromatography. Samples from left to right are (1) protein ladder (2) WT, (3) Y838F, (4) R888A, (5) R888K, (6) D146A, (7) GAA, (8) D146A+GAA. [B] The cartoon depicts de novo initiation/elongation on the DENV 2 minireplicon ${ }^{21}$ (left) and elongation from a primed template (right). The de novo initiation/elongation and elongation polymerase activities of NS5 mutants Y838F, R888A, R888K, D146A, GAA and D146A+GAA are compared with WT NS5 which was normalized to $100 \%$. The data is presented as average \pm SD from two to four independent repeat experiments. The statistical significance of difference between two groups was evaluated by Student t-test, and a $P$ value $<0.05$ was considered significant $(<=0.0001,<=0.001,<=$ $0.01,<=0.05$ were represented by ${ }^{* * *},{ }^{* * *},{ }^{* *},{ }^{*}$, respectively).

Figure 4. Analysis of DENV2 NS5 binding to 5' SLA or 3'SL RNA [A] Schematic illustration of DENV2 genome showing the position, sequence and size of $5^{\prime}$ terminal stemloop A (5' SLA) and 3' terminal stem-loop (3'SL) within the 5' and 3' untranslated regions (UTRs). Purity of the in vitro synthesized and purified 5' SLA and 3'SL RNA samples were analyzed by agarose gel electrophoresis. Secondary structures of 5'SLA and 3'SL were predicted by mfold web server ${ }^{42}$ with the thermodynamic free energy $\triangle G$ of the folding process at $37^{\circ} \mathrm{C}$ indicated. [B] \& [C] RNA electrophoretic mobility shift assay (REMSA) for 5' SLA [B] or 3' SL [C] with WT NS5 or NS5 R888A mutant, analyzed on $1.2 \%$ agarose gel with lane number indicated at the bottom. Lane 1 in each of the four gels represented 5' SLA [B] or 3' SL [C] RNA alone in binding buffer. Lane 2-7 represented binding reactions of NS5 with RNA at molar ratio of $0: 1,0.5: 1,1: 1,2: 1,4: 1,8: 1$ and $16: 1$ as indicated on top of each lane. [D] Binding affinity of 5' SLA or 3' SL with WT NS5 or NS5 mutants Y838F, R888A, D146A, 
GAA, D146A+GAA reflected by apparent dissociation constant $(\mathrm{Kd})$ was calculated from band intensities using ImageJ from two to four independent repeat experiments represented as average \pm SD. The statistical significance of difference between two groups was evaluated by Student t-test, and a $P$ value $<0.05$ was considered significant $(<=0.0001$, $<=0.001,<=0.01,<=0.05$ were represented by ${ }^{* * *},{ }^{* * *},{ }^{* *},{ }^{*}$, respectively).

Figure 5. Western blot analysis of WT NS5 binding to biotinylated 5'SLA RNA following Co-immunoprecipitation. [A] WT NS5 was incubated with immobilized biotinylated 5'SLA. After excess unbound NS5 was removed, increasing amounts of 3'SL were added (left). Quantitation of the NS5 band was performed using ImageJ and normalized to $100 \%$ for the sample with no added 3'SL (right). [B] WT NS5 was incubated with immobilized biotinylated 5'SLA as in [A], but increasing amounts of 3'SL was added without removal of excess unbound NS5 (left). Quantitation of the NS5 was done as in [A] (right).

Molar ratio of 3'SL to 5'SLA is shown on top of the gel image, and NS5 was detected using the 5M1 antibody. The statistical significance of difference between two groups was evaluated by Student t-test, and a $P$ value $<0.05$ was considered significant $(<=0.0001$, $<=0.001,<=0.01,<=0.05$ were represented by ${ }^{* * *},{ }^{* \star *},{ }^{* *},{ }^{*}$, respectively).

Figure 6. Analysis of 3'SL RNA interaction with WT and mutant NS5 by coimmunoprecipitation and Dynamic Light Scattering. [A] Western blot analysis showing pull-down of WT NS5, R888A mutant and GAA mutant through 3'SL RNA immobilized on streptavidin Sepharose beads or the "no-RNA" mock coupled Sepharose beads as control. NS5 was detected by in-house 5M1 antibody(ref) which recognizes the MTase domain. [B] Quantification of pull-down WT, R888A and GAA with the amount of WT normalized to $100 \%$. The data were represented as average \pm SD from two independent repeat experiments. The statistical significance of difference between two groups was evaluated by Student t-test, and a $P$ value $<0.05$ was considered significant $(<=0.0001,<=0.001,<=0.01,<=0.05$ were represented by ${ }^{* * * *},{ }^{* * *},{ }^{* *},{ }^{*}$, respectively). [C] \& [D] Intensity-based size distribution profiles from Dynamic light scattering (DLS) experiments showing 3'SL RNA, NS5 WT or molar ratio 1:1 mixture of NS5 and 3'SL complex [C] or R888A mutant [D] and the molar ratio 1:1 mixture of NS5 R888 and 3'SL. The DLS experiments were repeated twice with similar size distribution profiles for each sample.

Figure 7. Analysis of DENV2 NS5 binding to 3'SL RNA and 3'SL RNA with top-loop or side-loop deletion. [A] Secondary structures of 3'SL RNA and 3'SL with deleted top lop (3'SL-TL ${ }^{\text {del }}$ ) or side loop (3'SL-SL ${ }^{\text {del }}$ ) were predicted by mfold web server with folding free energy $\Delta G$ at $37^{\circ} \mathrm{C}$ shown in below. Corresponding RNA samples produced by in vitro transcription followed by purification were analyzed by agarose gel electrophoresis. [B] \& [C] RNA electrophoretic mobility shift assay (REMSA) for NS5 WT [B] or R888A mutant [C] with 3' SL, 3'SL-TL ${ }^{\text {del }}$ or 3'SL-SL ${ }^{\text {del }}$ analyzed on $1.2 \%$ agarose gel with lane number indicated at the bottom. Lane 2, 8, and 15 in both gels represented 3'SL, 3'SL-TL ${ }^{\text {del }}, 3$ 'SL-SL ${ }^{\text {del }}$ RNA alone in binding buffer. Lane 3-7, 9-13 and 15-19 represented binding reactions of NS5 with RNA at molar ratio of $0: 1,1: 1,2: 1,4: 1,8: 1$ and $16: 1$ as indicated on top of each lane. The REMSA experiments were performed three times with similar results. [D] Binding affinity of WT NS5 or R888A mutant with 3' SL, 3'SL-TL del or 3'SL-SL ${ }^{\text {del }}$ reflected by apparent dissociation constant $(\mathrm{Kd})$ was calculated from three independent repeat experiments represented as average \pm SD. The statistical significance of difference between two groups 
was evaluated by Student t-test, and a $P$ value $<0.05$ was considered significant $(<=$ $0.0001,<=0.001,<=0.01,<=0.05$ were represented by ${ }^{* \star \star \star},{ }^{* \star \star},{ }^{\star \star},{ }^{*}$, respectively).

Figure 8. A mechanistic model of de novo initiation and elongation of flavivirus genomic RNA, highlighting the critical events of genome circularization, NS5 dimerization, and UFSmediated de novo initiation and elongation (see text for details). NS5 (green) bound to 5' UTR (SLA and SLB) is well established by Gamarnik group ${ }^{5-7}$ and the present study showed the specific interaction between a molecule of NS5 (purple) through R888 at its C-terminal region and the top-loop of 3'SL. Long range RNA interactions within the open reading frame region ${ }^{49}$ juxtaposes the 5 and 3' CS and DAR for RNA cyclization supported by NS5-RNA complex formation. The cis-acting tunable UFS region ensures primer-independent RNA replication by serving as a gate keeper to ensure formation of a productive stable de novo initiation complex that can carry out processive RNA replication in concert with viral and host proteins.

\section{Acknowledgments}

Financial support in part from the National Research Foundation (NRF2016CRP001-063) and National Medical Research Council of Singapore (NMRC grant $\mathrm{MOH}-000086$ : MOH-OFIRG18-may-0006) is gratefully acknowledged.

\section{Author contributions}

Author contributions: S.W., K.W.K.C., J.K.F and S.G.V. conceived the study. K.W.K.C. and S.W performed and analyzed the in vitro infection studies in cell lines. S.W. and M.J.A.T. performed and analyzed the biochemical and RNA interaction studies. M.J.A.T. and C.F. contributed RNA and protein reagents used in the study. S.W. D.L. J.L. and J.F.K carried out the DLS and structural analysis; S.W., M.J.A.T.and S.G.V. wrote the manuscript. All authors read and commented on the final manuscript.

\section{Competing interests.}

Authors report no competing interests.

\section{Materials \& Correspondence.}

Correspondence and material requests should be addressed. Subhash G. Vasudevan (subhash.vasudevan@duke-nus.edu.sg)

\section{REFERENCES}

1 Gubler, D. J. Dengue and dengue hemorrhagic fever. Clinical microbiology reviews 11, 480-496 (1998).

2 Thomas, S. J. \& Yoon, I.-K. A review of Dengvaxia®: development to deployment. Hum Vaccin Immunother 15, 2295-2314, doi:10.1080/21645515.2019.1658503 (2019). 

The $5^{\prime}$ and $3^{\prime}$ Untranslated Regions of the Flaviviral Genome. Viruses 9 , doi:10.3390/v9060137 (2017).

4 J. Barrows, N. et al. Biochemistry and Molecular Biology of Flaviviruses. Vol. 118 (2018).

5 Lodeiro, M. F., Filomatori, C. V. \& Gamarnik, A. V. Structural and functional studies of the promoter element for dengue virus RNA replication. Journal of virology 83, 993-1008, doi:10.1128/JVI.01647-08 (2009).

6 Filomatori, C. V., Iglesias, N. G., Villordo, S. M., Alvarez, D. E. \& Gamarnik, A. V. RNA Sequences and Structures Required for the Recruitment and Activity of the Dengue Virus Polymerase. Journal of Biological Chemistry 286, 69296939, doi:10.1074/jbc.M110.162289 (2011).

7 Filomatori, C. V. et al. A 5' RNA element promotes dengue virus RNA synthesis on a circular genome. Genes \& development 20, 2238-2249, doi:10.1101/gad.1444206 (2006).

8 Lee, E. et al. Structures of flavivirus RNA promoters suggest two binding modes with NS5 polymerase. Nature Communications 12, 2530, doi:10.1038/s41467-021-22846-1 (2021).

9 Liu, Z.-Y. et al. Viral RNA switch mediates the dynamic control of flavivirus replicase recruitment by genome cyclization. eLife 5, e17636, doi:10.7554/eLife.17636 (2016).

10 Alvarez, D. E., De Lella Ezcurra, A. L., Fucito, S. \& Gamarnik, A. V. Role of RNA structures present at the 3'UTR of dengue virus on translation, RNA synthesis, and viral replication. Virology 339, 200-212, doi:10.1016/j.virol.2005.06.009 (2005).

11 Chapman, E. G. et al. The structural basis of pathogenic subgenomic flavivirus RNA (sfRNA) production. Science (New York, N.Y.) 344, 307-310, doi:10.1126/science.1250897 (2014).

12 Clarke, B. D., Roby, J. A., Slonchak, A. \& Khromykh, A. A. Functional noncoding RNAs derived from the flavivirus $3^{\prime}$ untranslated region. Virus Research 206, 53-61, doi:https://doi.org/10.1016/j.virusres.2015.01.026 (2015).

13 Akiyama, B. M. et al. Zika virus produces noncoding RNAs using a multipseudoknot structure that confounds a cellular exonuclease. Science (New York, N.Y.) 354, 1148-1152, doi:10.1126/science.aah3963 (2016).

14 Slonchak, A. \& Khromykh, A. A. Subgenomic flaviviral RNAs: What do we know after the first decade of research. Antiviral research 159, 13-25, doi:https://doi.org/10.1016/i.antiviral.2018.09.006 (2018).

15 Hodge, K. et al. Identification of a Conserved RNA-dependent RNA Polymerase (RdRp)-RNA Interface Required for Flaviviral Replication. J Biol Chem 291, 17437-17449, doi:10.1074/jbc.M116.724013 (2016).

16 Tilgner, M., Deas, T. S. \& Shi, P.-Y. The flavivirus-conserved penta-nucleotide in the $3^{\prime}$ stem-loop of the West Nile virus genome requires a specific sequence and structure for RNA synthesis, but not for viral translation. Virology 331, 375-386, doi:https://doi.org/10.1016/i.virol.2004.07.022 (2005).

17 Villordo, S. M. \& Gamarnik, A. V. Genome cyclization as strategy for flavivirus RNA replication. Virus Res 139, 230-239, doi:10.1016/j.virusres.2008.07.016 (2009). 
18 Dong, H. et al. 2'-O methylation of internal adenosine by flavivirus NS5 methyltransferase. PLoS pathogens 8, e1002642, doi:10.1371/journal.ppat.1002642 (2012).

19 Saw, W. G. et al. Structural insight and flexible features of NS5 proteins from all four serotypes of Dengue virus in solution. Acta Crystallogr D Biol Crystallogr 71, 2309-2327, doi:10.1107/S1399004715017721 (2015).

20 Ashour, J., Laurent-Rolle, M., Shi, P.-Y. \& García-Sastre, A. NS5 of Dengue Virus Mediates STAT2 Binding and Degradation. Journal of Virology 83, 5408, doi:10.1128/JVI.02188-08 (2009).

21 Zhao, Y. et al. Flexibility of NS5 Methyltransferase-Polymerase Linker Region Is Essential for Dengue Virus Replication. Journal of Virology 89, 10717, doi:10.1128/JVI.01239-15 (2015).

22 Zhao, Y. et al. A crystal structure of the Dengue virus NS5 protein reveals a novel inter-domain interface essential for protein flexibility and virus replication. PLoS pathogens 11, e1004682, doi:10.1371/journal.ppat.1004682 (2015).

23 Subramanian Manimekalai, M. S., Saw, W. G., Pan, A., Grüber, A. \& Grüber, $G$. Identification of the critical linker residues conferring differences in the compactness of NS5 from Dengue virus serotype 4 and NS5 from Dengue virus serotypes 1-3. Acta Crystallographica Section D 72, 795-807, doi:10.1107/S2059798316006665 (2016).

24 Chang, D. C. et al. Evasion of early innate immune response by 2'-Omethylation of dengue genomic RNA. Virology 499, 259-266, doi:10.1016/j.virol.2016.09.022 (2016).

25 Lescar, J. et al. The Dengue Virus Replication Complex: From RNA Replication to Protein-Protein Interactions to Evasion of Innate Immunity. Advances in experimental medicine and biology 1062, 115-129, doi:10.1007/978-981-10-8727-1_9 (2018).

26 Brand, C., Bisaillon, M. \& Geiss, B. J. Organization of the Flavivirus RNA replicase complex. WIREs RNA 8, e1437, doi:https://doi.org/10.1002/wrna.1437 (2017).

27 Klema, V. J., Padmanabhan, R. \& Choi, K. H. Flaviviral Replication Complex: Coordination between RNA Synthesis and 5'-RNA Capping. Viruses 7, 46404656, doi:10.3390/v7082837 (2015).

28 Welsch, S. et al. Composition and Three-Dimensional Architecture of the Dengue Virus Replication and Assembly Sites. Cell Host \& Microbe 5, 365375, doi:https://doi.org/10.1016/j.chom.2009.03.007 (2009).

29 Cerikan, B. et al. A Non-Replicative Role of the 3' Terminal Sequence of the Dengue Virus Genome in Membranous Replication Organelle Formation. Cell Reports 32, 107859, doi:https://doi.org/10.1016/i.celrep.2020.107859 (2020).

30 Pryor, M. J. et al. Nuclear localization of dengue virus nonstructural protein 5 through its importin alpha/beta-recognized nuclear localization sequences is integral to viral infection. Traffic 8, 795-807, doi:10.1111/j.16000854.2007.00579.x (2007).

31 Hannemann, H. et al. Serotype-specific differences in dengue virus nonstructural protein 5 nuclear localization. J Biol Chem 288, 22621-22635, doi:10.1074/jbc.M113.481382 (2013).

32 Kumar, A. et al. Nuclear Localization of Dengue Virus Nonstructural Protein 5 Does Not Strictly Correlate with Efficient Viral RNA Replication and Inhibition of Type I Interferon Signaling. Journal of Virology 87, 4545, doi:10.1128/JVI.03083-12 (2013). 
33 Tay, M. Y. F. et al. Nuclear localization of dengue virus (DENV) 1-4 nonstructural protein 5; protection against all 4 DENV serotypes by the inhibitor Ivermectin. Antiviral Research 99, 301-306, doi:https://doi.org/10.1016/j.antiviral.2013.06.002 (2013).

34 Tay, M. Y. F. et al. The C-terminal 18 Amino Acid Region of Dengue Virus NS5 Regulates its Subcellular Localization and Contains a Conserved Arginine Residue Essential for Infectious Virus Production. PLoS pathogens 12, e1005886, doi:10.1371/journal.ppat.1005886 (2016). Klema, V. J. et al. Dengue Virus Nonstructural Protein 5 (NS5) Assembles into a Dimer with a Unique Methyltransferase and Polymerase Interface. PLoS pathogens 12, e1005451, doi:10.1371/journal.ppat.1005451 (2016).

36 Saw, W. G., Chan, K. W., Vasudevan, S. G. \& Gruber, G. Zika virus nonstructural protein 5 residue $\mathrm{R} 681$ is critical for dimer formation and enzymatic activity. FEBS letters 593, 1272-1291, doi:10.1002/18733468.13437 (2019).

37 Yap, T. L. et al. Crystal structure of the dengue virus RNA-dependent RNA polymerase catalytic domain at 1.85-angstrom resolution. Journal of virology 81, 4753-4765, doi:10.1128/JVI.02283-06 (2007).

38 Bujalowski, P. J., Bujalowski, W. \& Choi, K. H. Identification of the viral RNA promoter stem loop A (SLA)-binding site on Zika virus polymerase NS5. Scientific Reports 10, 13306, doi:10.1038/s41598-020-70094-y (2020).

39 Tay, M. Y. F. et al. The C-terminal 50 amino acid residues of dengue NS3 protein are important for NS3-NS5 interaction and viral replication. The Journal of biological chemistry 290, 2379-2394, doi:10.1074/jbc.M114.607341 (2015).

40 Zhao, Y. et al. Molecular basis for specific viral RNA recognition and 2'-Oribose methylation by the dengue virus nonstructural protein 5 (NS5). Proceedings of the National Academy of Sciences 112, 14834, doi:10.1073/pnas.1514978112 (2015).

41 Potisopon, S. et al. The methyltransferase domain of dengue virus protein NS5 ensures efficient RNA synthesis initiation and elongation by the polymerase domain. Nucleic acids research 42, 11642-11656, doi:10.1093/nar/gku666 (2014).

42 Zuker, M. Mfold web server for nucleic acid folding and hybridization prediction. Nucleic acids research 31, 3406-3415 (2003).

43 Wang, S. et al. Subgenomic RNA from Dengue Virus Type 2 Suppresses Replication of Dengue Virus Genomes and Interacts with Virus-Encoded NS3 and NS5 Proteins. ACS Infectious Diseases 6, 436-446, doi:10.1021/acsinfecdis.9b00384 (2020).

44 Schindelin, J., Rueden, C. T., Hiner, M. C. \& Eliceiri, K. W. The ImageJ ecosystem: An open platform for biomedical image analysis. Molecular Reproduction and Development 82, 518-529, doi:https://doi.org/10.1002/mrd.22489 (2015).

45 Zhao, Y. et al. Identification and molecular characterization of human antibody fragments specific for dengue NS5 protein. Virus Research 179, 225-230, doi:https://doi.org/10.1016/j.virusres.2013.11.010 (2014).

46 Stetefeld, J., McKenna, S. A. \& Patel, T. R. Dynamic light scattering: a practical guide and applications in biomedical sciences. Biophys Rev 8, 409427, doi:10.1007/s12551-016-0218-6 (2016). 
47 Alvarez, D. E., Lodeiro, M. F., Ludueña, S. J., Pietrasanta, L. I. \& Gamarnik, A. V. Long-range RNA-RNA interactions circularize the dengue virus genome. Journal of virology 79, 6631-6643, doi:10.1128/JVI.79.11.6631-6643.2005 (2005).

48 Clyde, K., Barrera, J. \& Harris, E. The capsid-coding region hairpin element (cHP) is a critical determinant of dengue virus and West Nile virus RNA synthesis. Virology 379, 314-323, doi:10.1016/j.virol.2008.06.034 (2008).

49 Huber, R. G. et al. Structure mapping of dengue and Zika viruses reveals functional long-range interactions. Nature Communications 10, 1408, doi:10.1038/s41467-019-09391-8 (2019).

50 Appleby, T. C. et al. Structural basis for RNA replication by the hepatitis C virus polymerase. Science (New York, N.Y.) 347, 771, doi:10.1126/science.1259210 (2015).

51 Selisko, B. et al. Molecular Basis for Nucleotide Conservation at the Ends of the Dengue Virus Genome. PLoS pathogens 8, e1002912, doi:10.1371/journal.ppat.1002912 (2012).

52 Johansson, M., Brooks, A. J., Jans, D. A. \& Vasudevan, S. G. A small region of the dengue virus-encoded RNA-dependent RNA polymerase, NS5, confers interaction with both the nuclear transport receptor importin-beta and the viral helicase, NS3. The Journal of general virology 82, 735-745, doi:10.1099/0022-1317-82-4-735 (2001).

53 Brooks, A. J., Johansson, M., Criswell, E., Jans, D. A. \& Vasudevan, S. G. The interdomain region of dengue NS5 protein interacts with NS3 and host proteins. (2002).

54 Zou, G. et al. Functional analysis of two cavities in flavivirus NS5 polymerase. The Journal of biological chemistry 286, 14362-14372, doi:10.1074/jbc.M110.214189 (2011).

55 Paradkar, Prasad N., Ooi, Eng E., Hanson, Brendon J., Gubler, Duane J. \& Vasudevan, Subhash G. Unfolded protein response (UPR) gene expression during antibody-dependent enhanced infection of cultured monocytes correlates with dengue disease severity. Bioscience Reports 31, 221, doi:10.1042/BSR20100078 (2011).

56 Collins, T. J. Image J for microscopy. Biotechniques 43, 25-30, doi:10.2144/000112517 (2007).

57 Doshi, K. J., Cannone, J. J., Cobaugh, C. W. \& Gutell, R. R. Evaluation of the suitability of free-energy minimization using nearest-neighbor energy parameters for RNA secondary structure prediction. BMC Bioinformatics 5, 105, doi:10.1186/1471-2105-5-105 (2004).

58 Wang, S. et al. Subgenomic RNA from Dengue Virus Type 2 Suppresses Replication of Dengue Virus Genomes and Interacts with Virus-Encoded NS3 and NS5 Proteins. ACS Infect Dis, doi:10.1021/acsinfecdis.9b00384 (2020). 
A

DENV2 WT

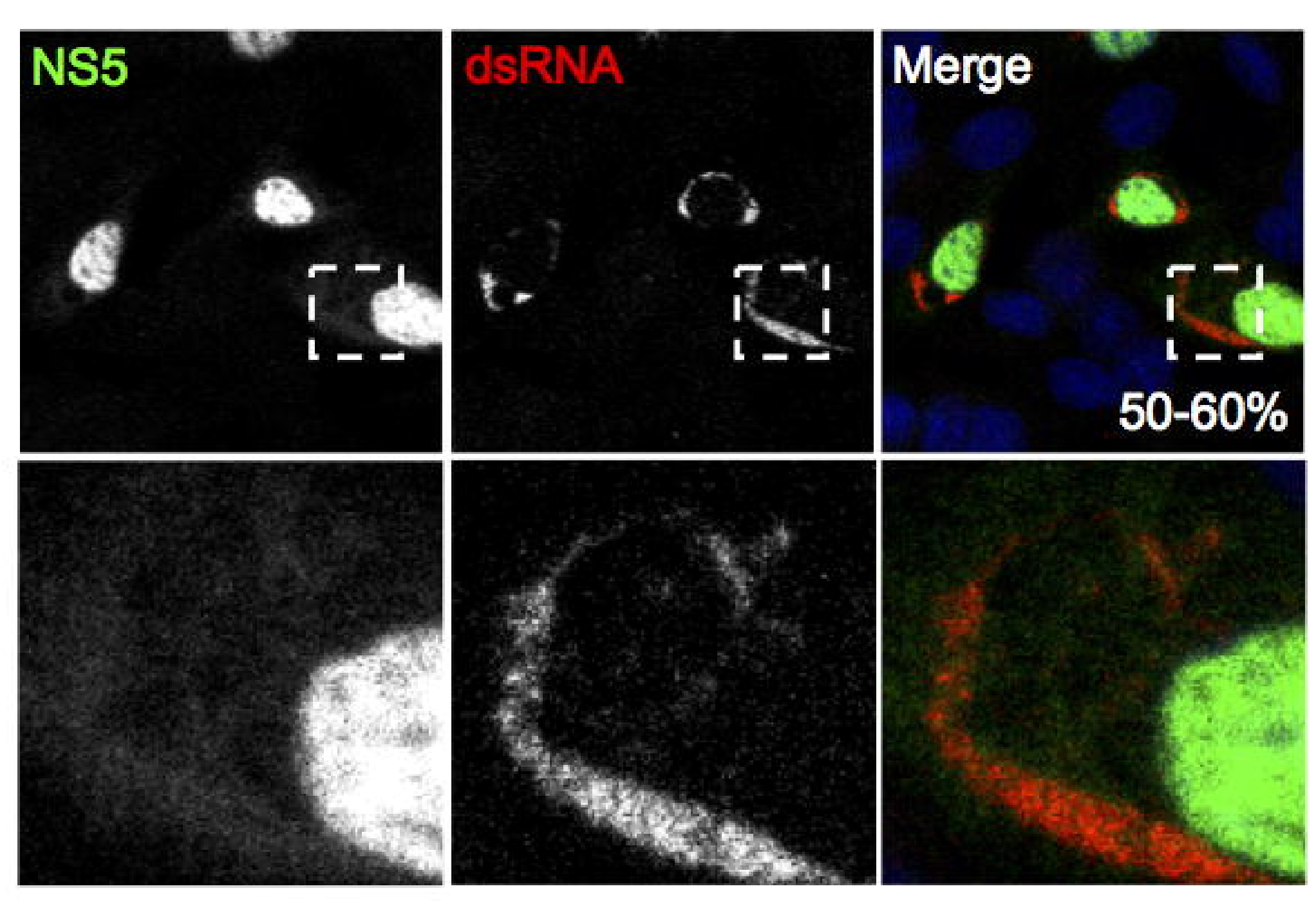

C

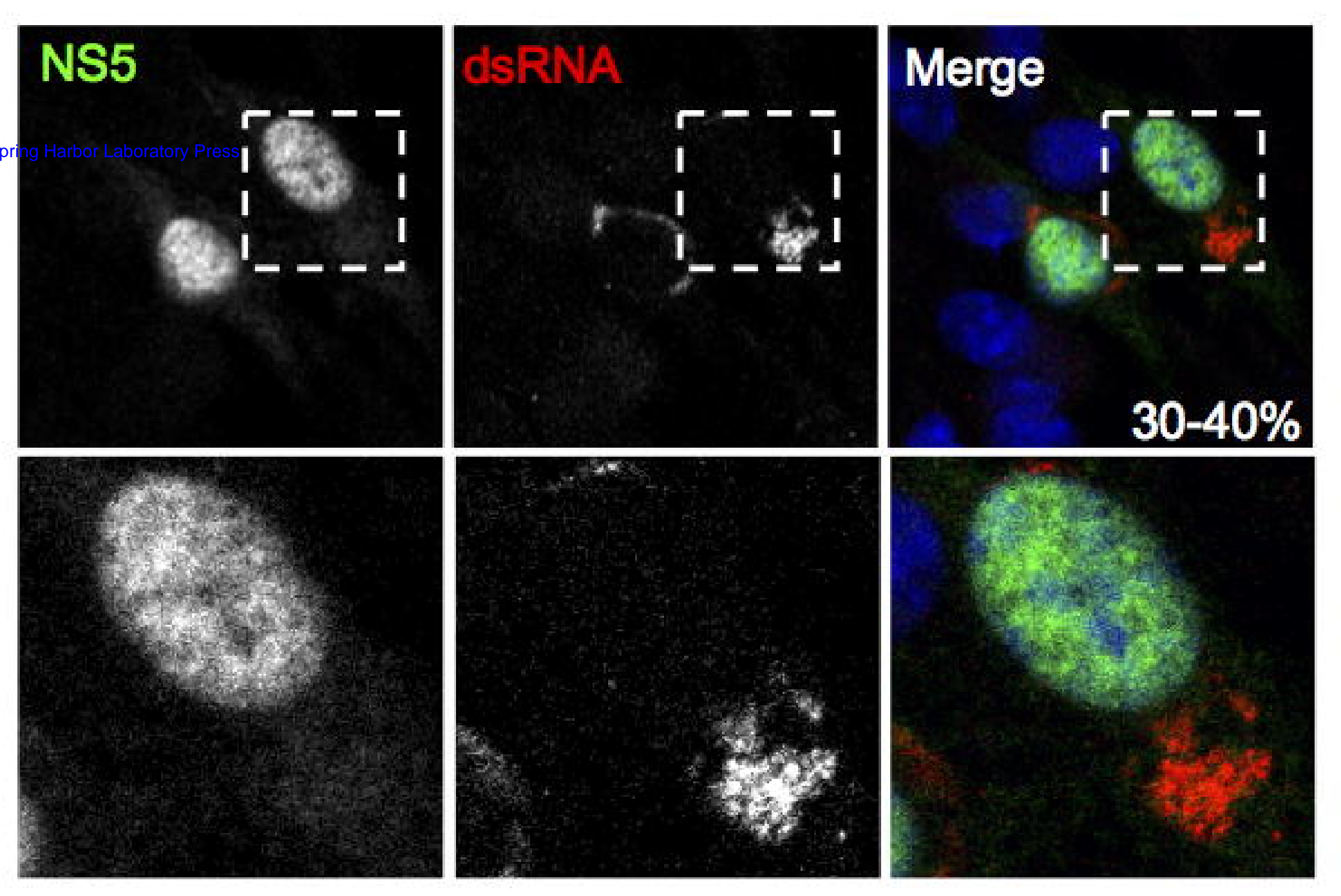

B

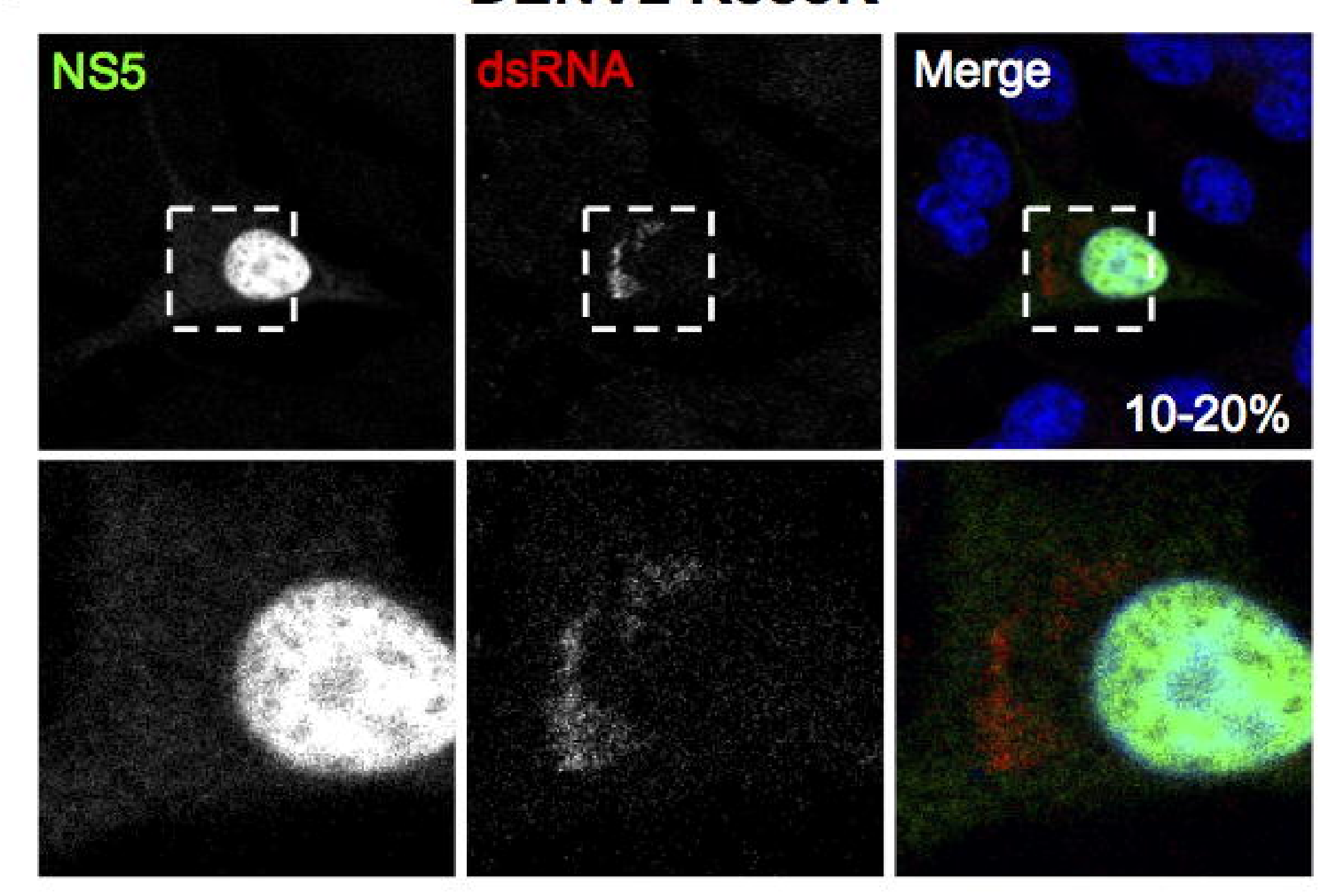

D

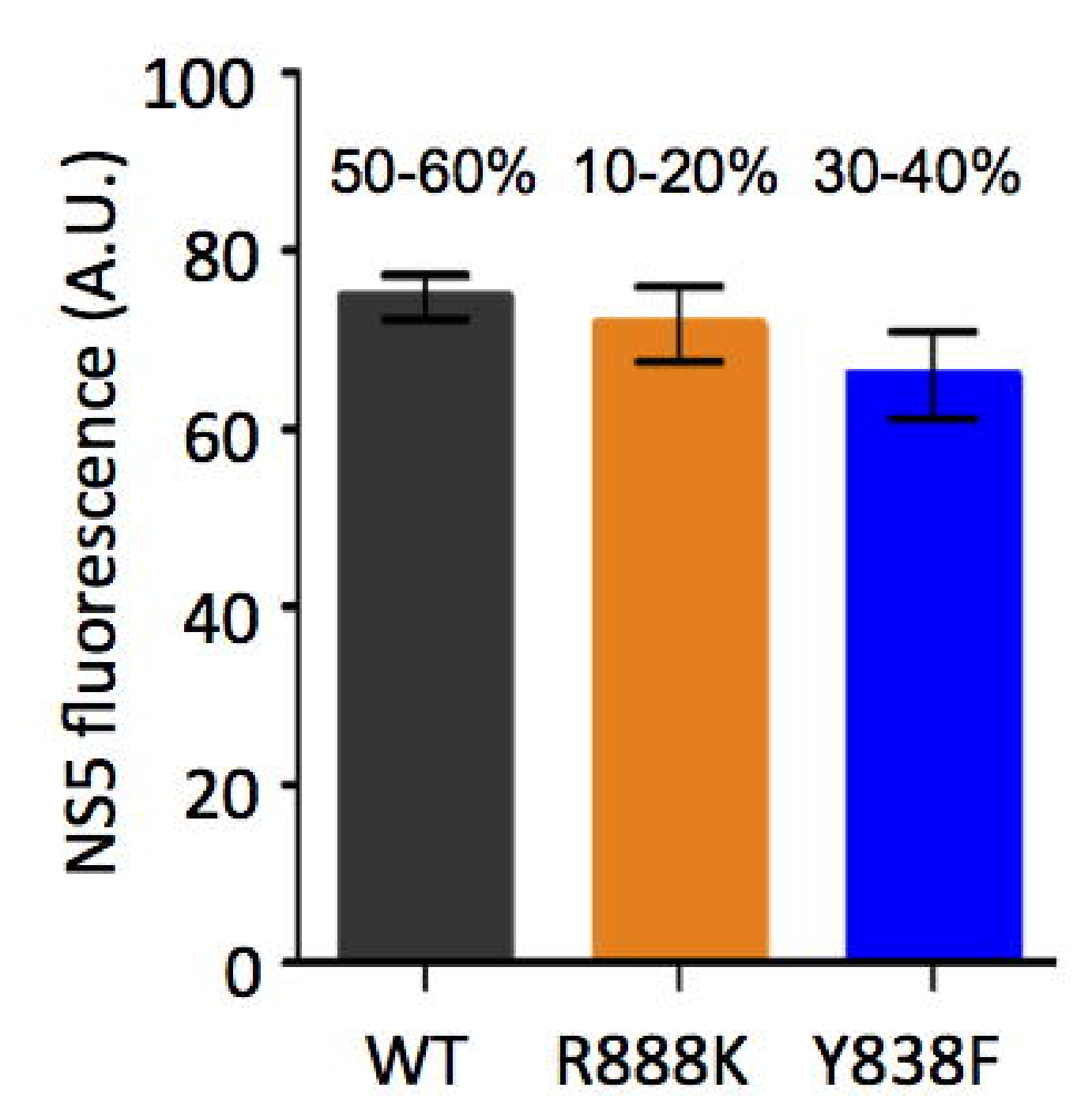

E

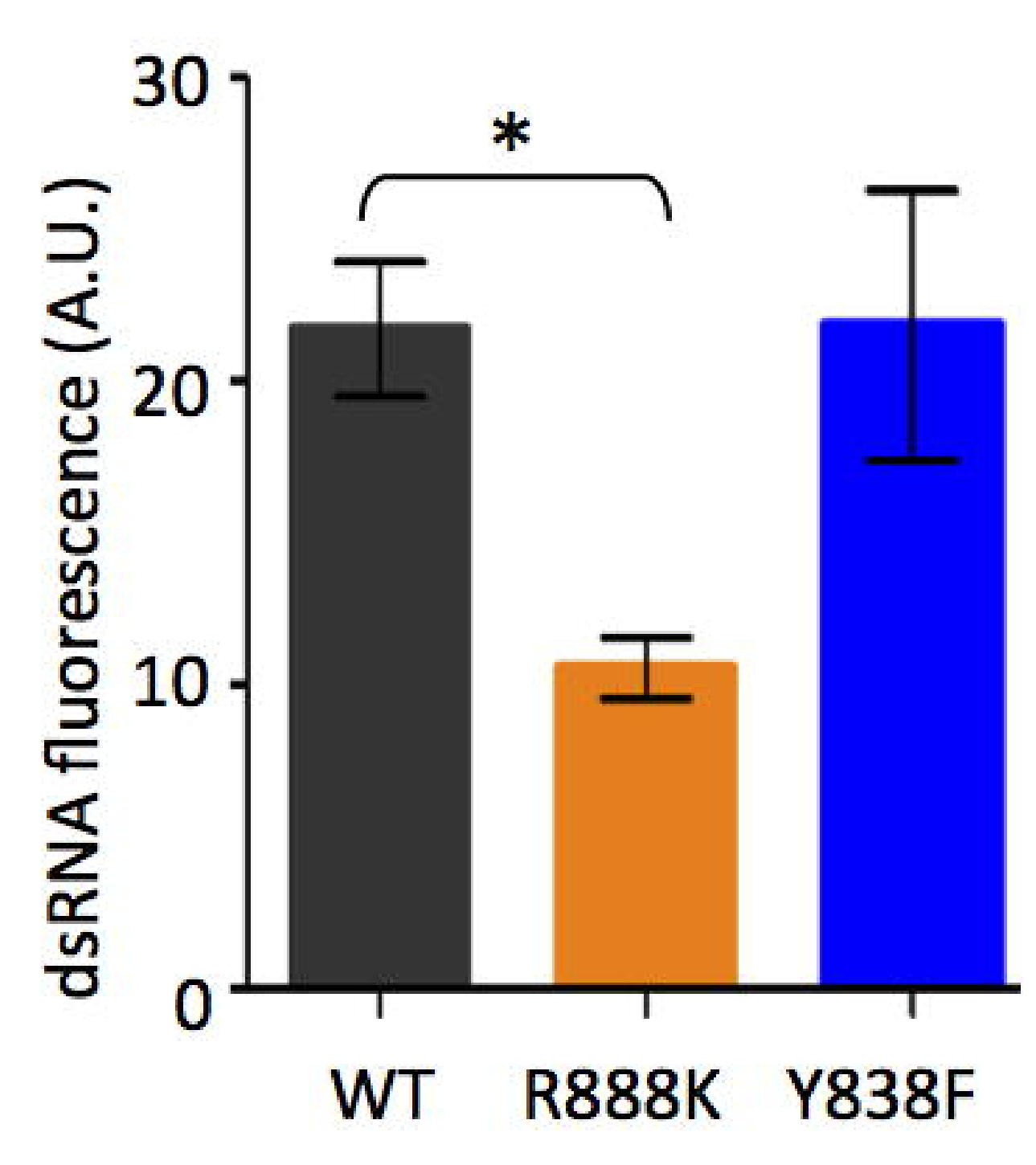



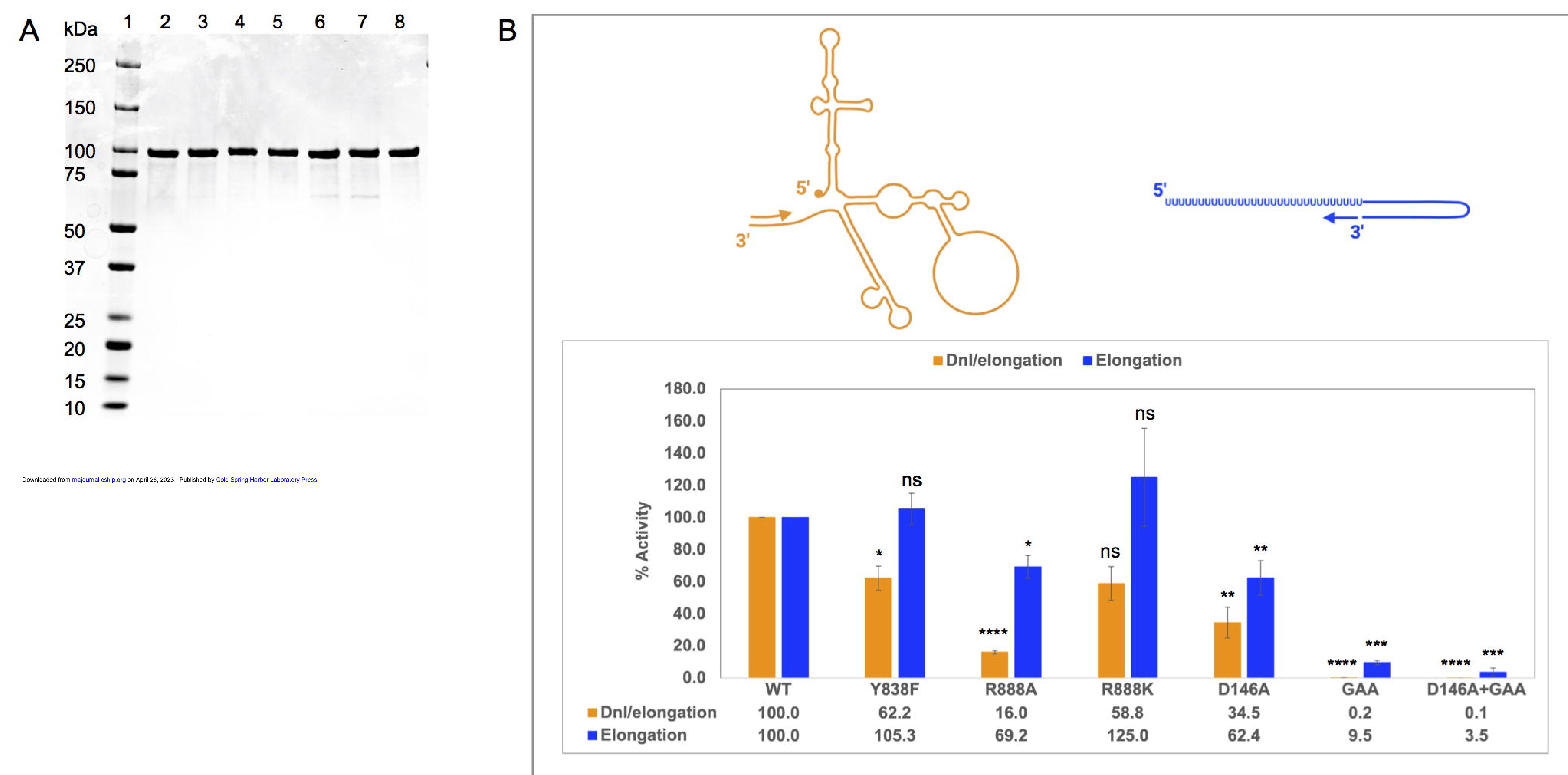


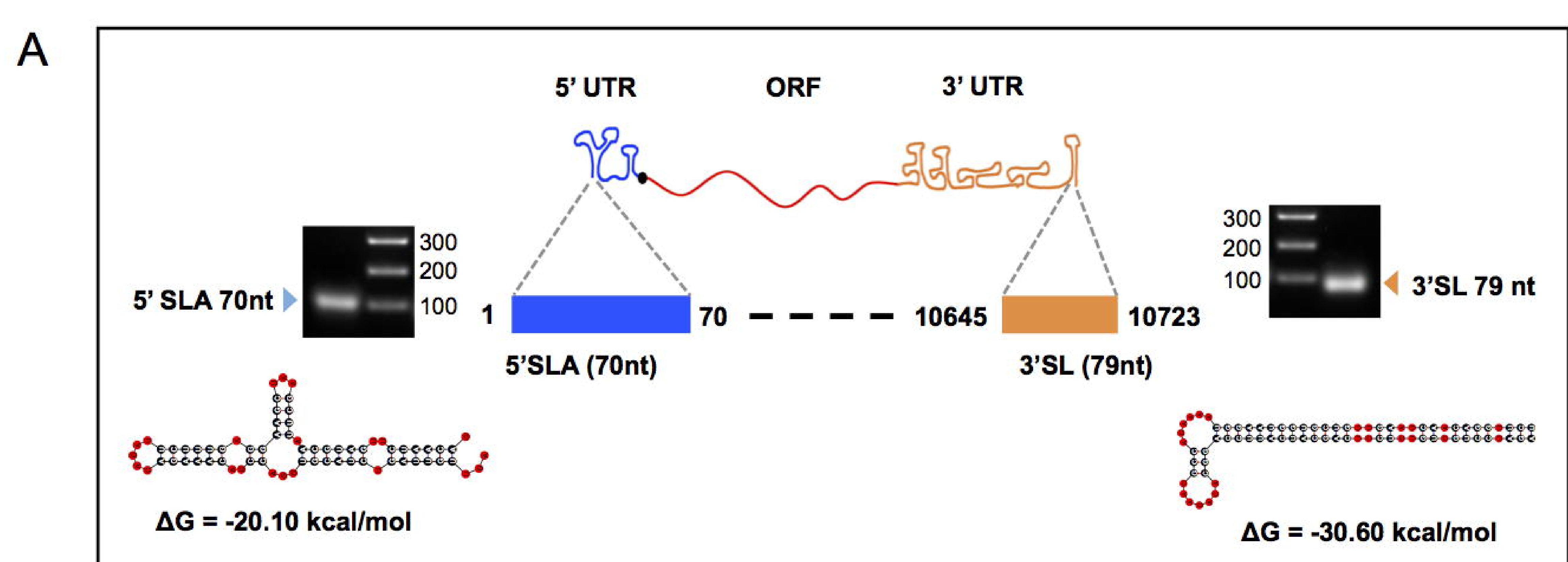

B

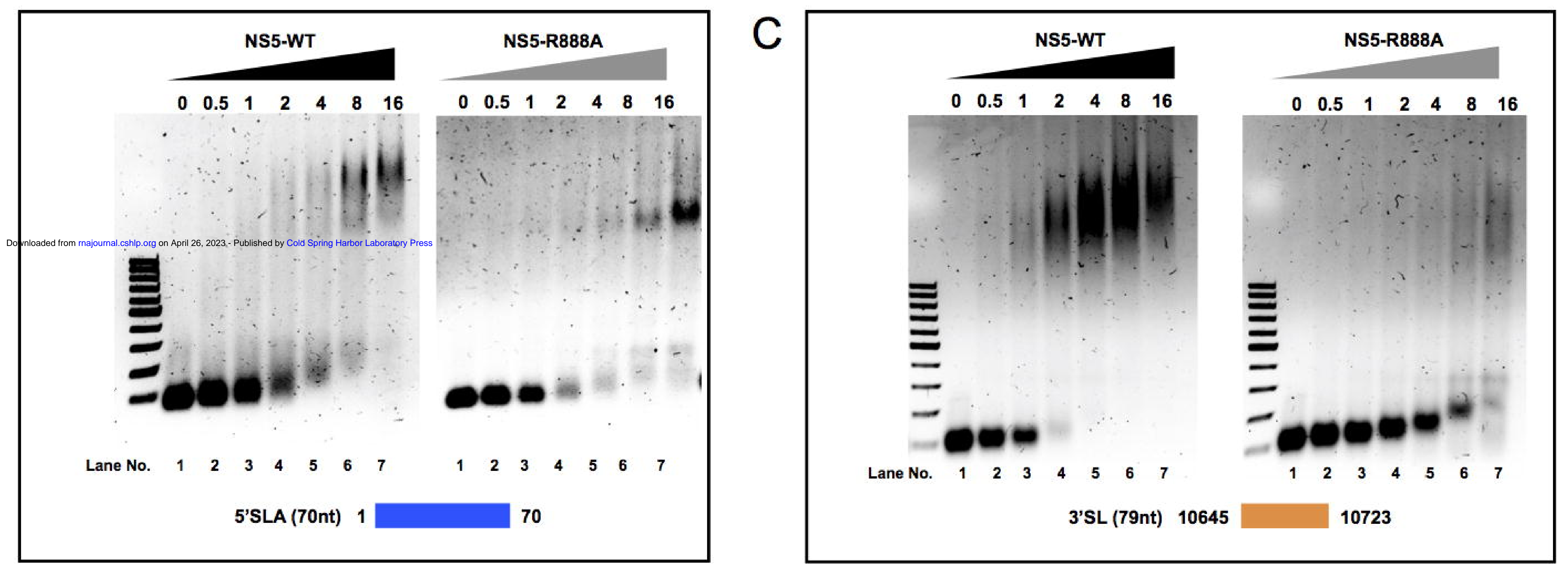

D

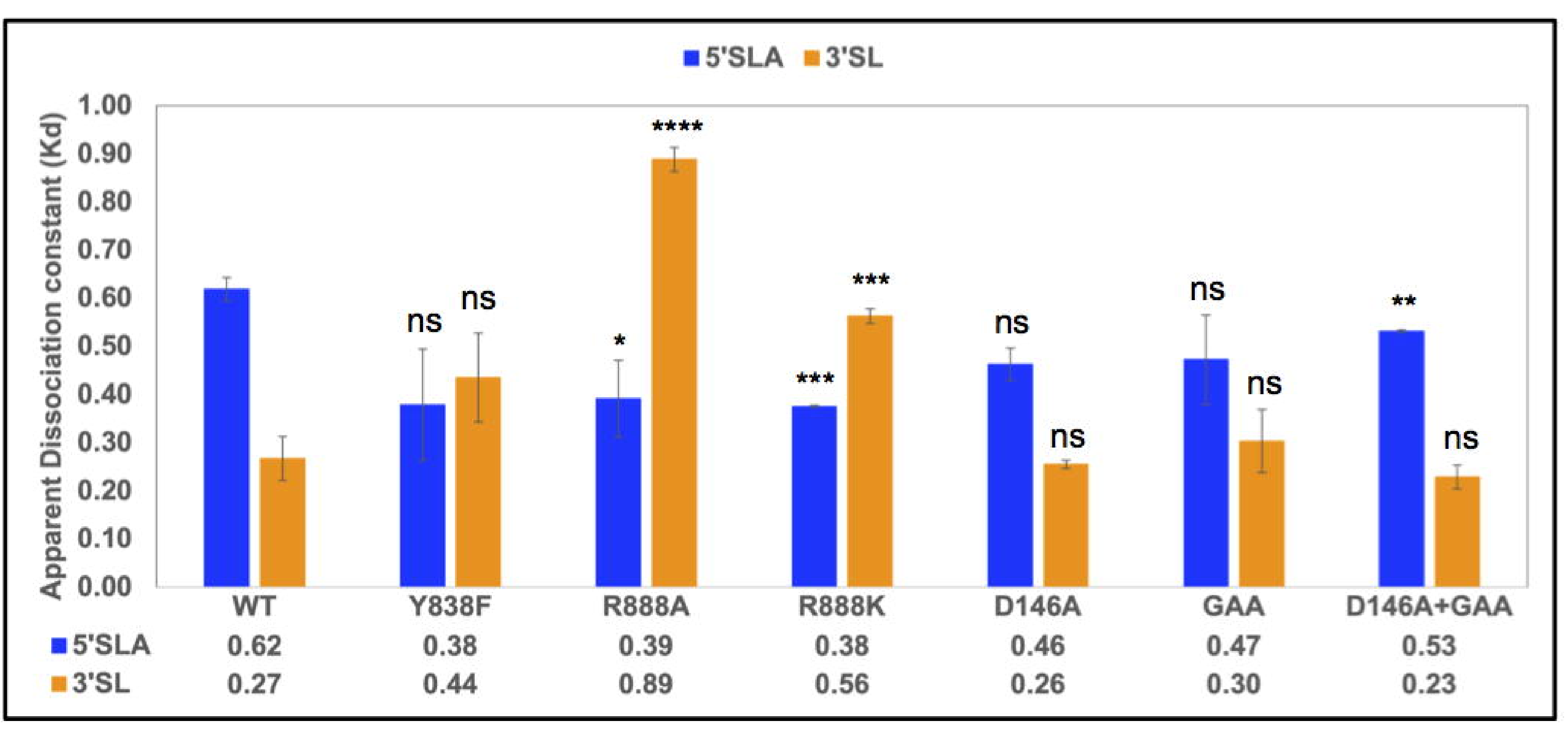


A

\section{No free NS5}

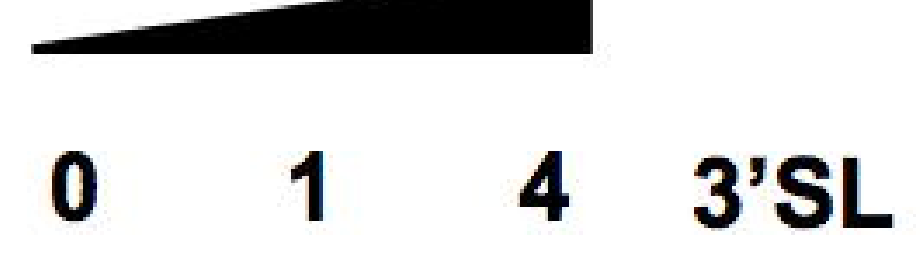

$+\quad+\quad+\quad$ 'SLA-biotin

$100 \mathrm{kDa}$

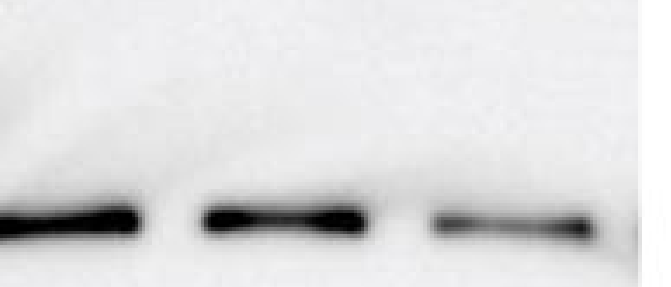

\section{B}

\section{Excess NS5}

$100 \mathrm{kDa}$ $\begin{array}{llllll}0 & 0.5 & 1 & 2 & 4 & 3\end{array}$

+++++5 'SLA-biotin
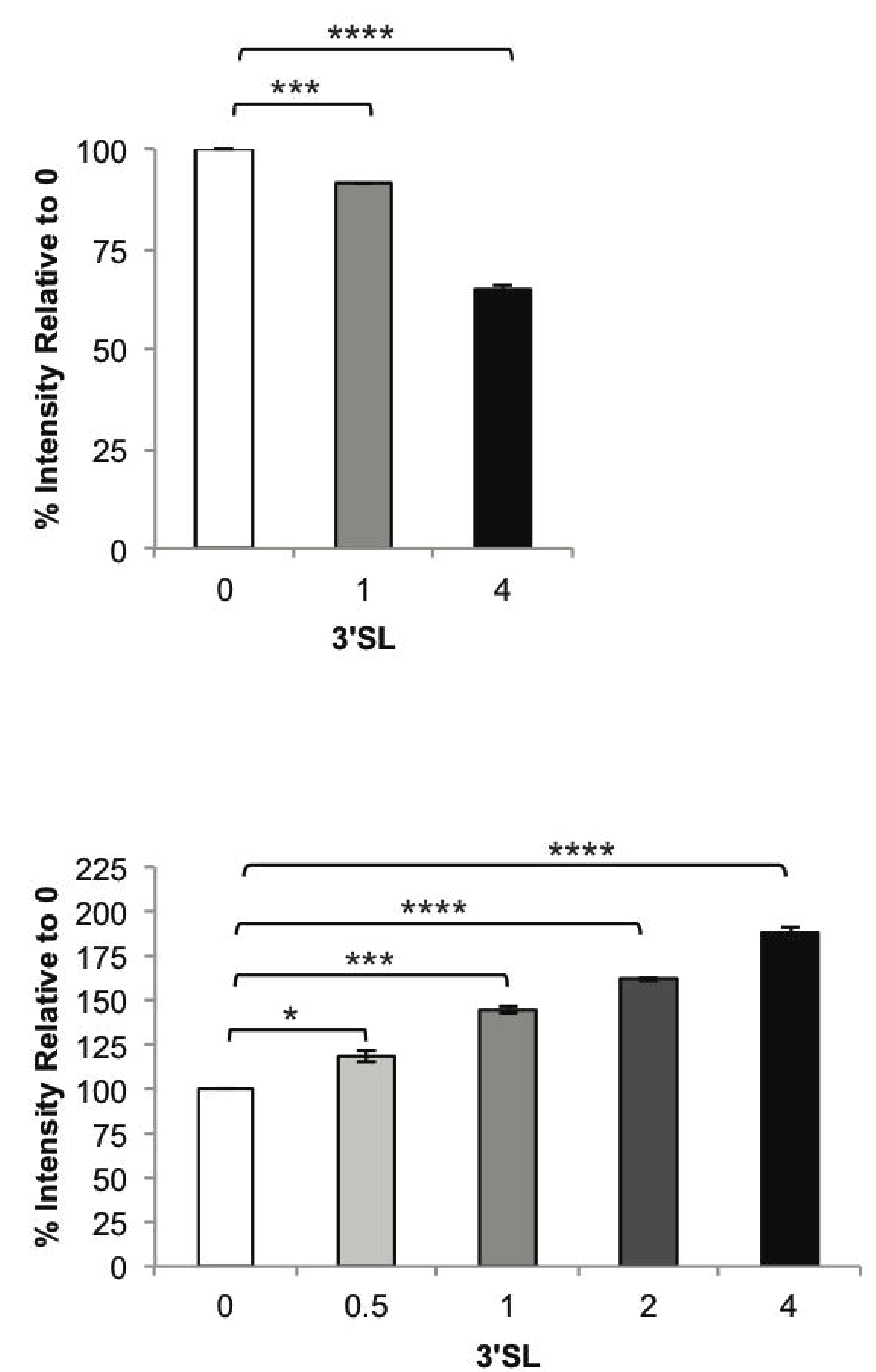


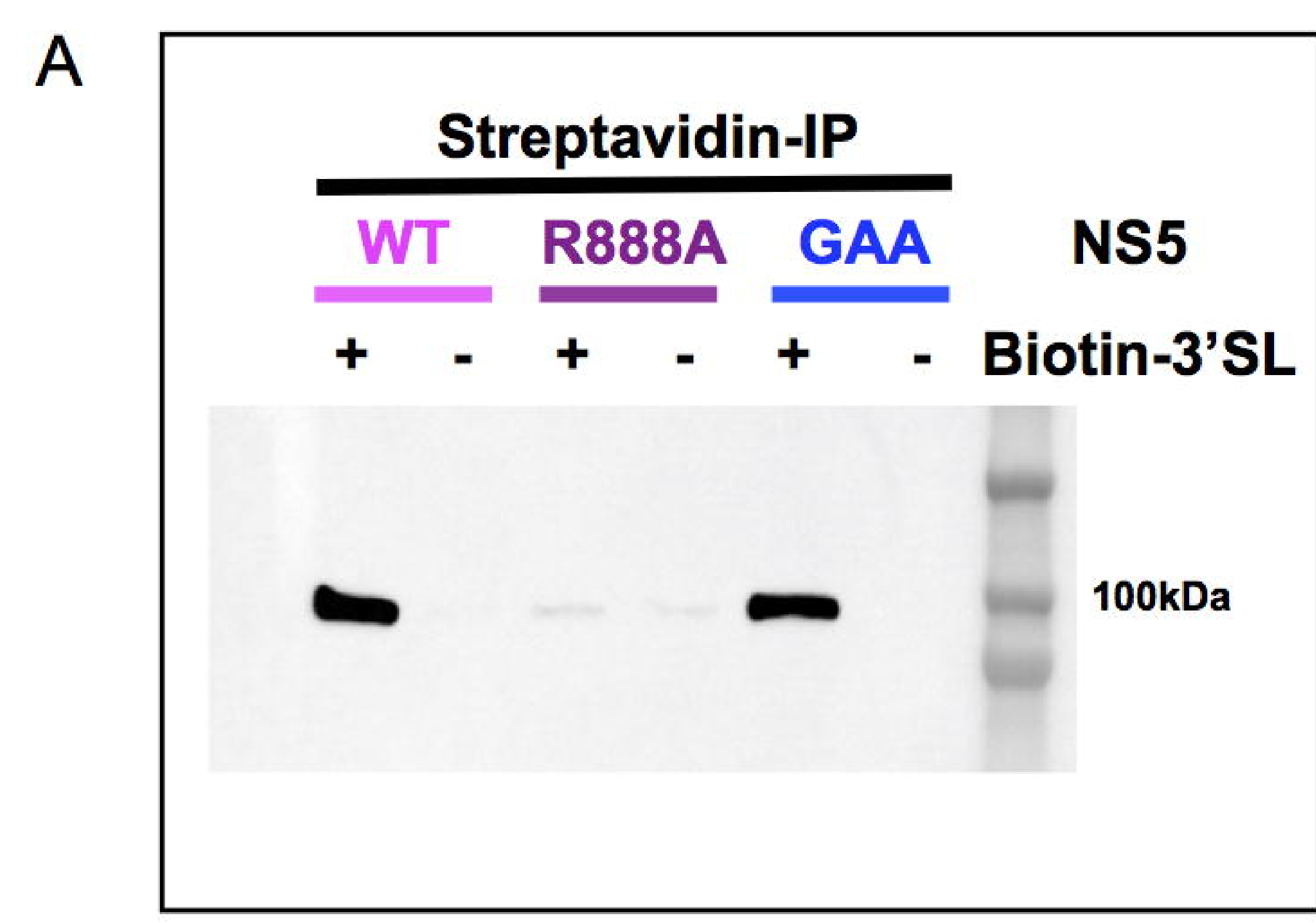

\section{B}

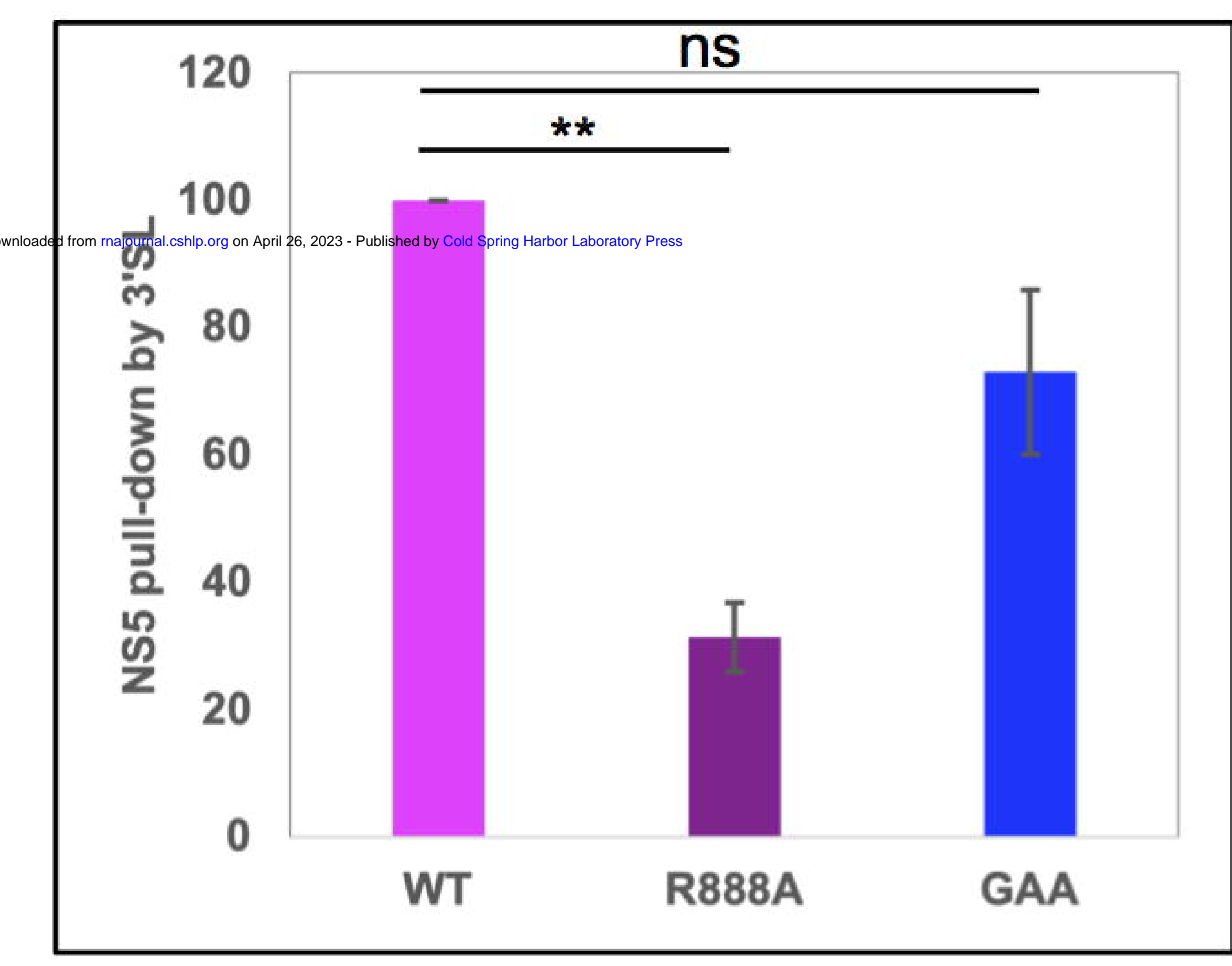

C

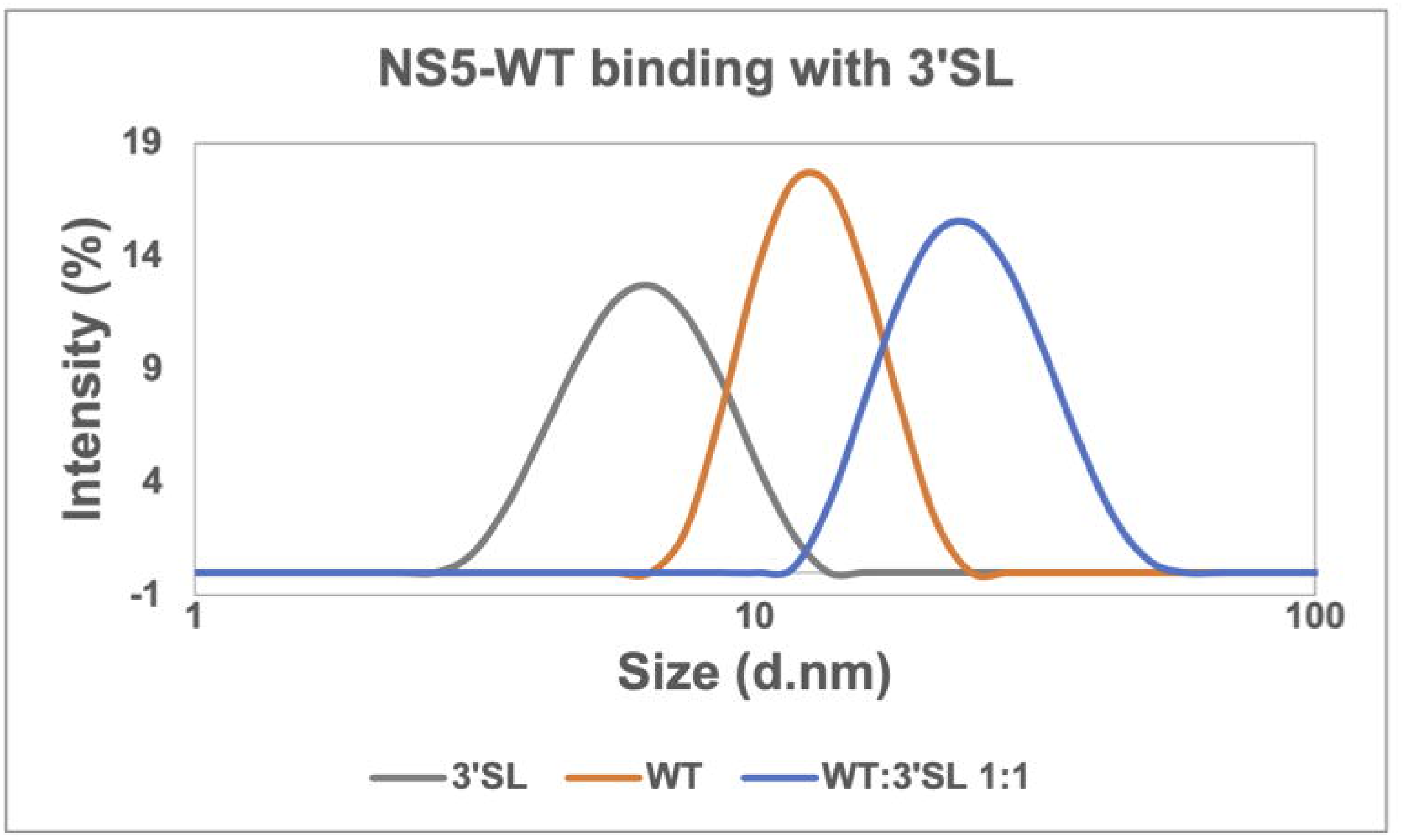

D

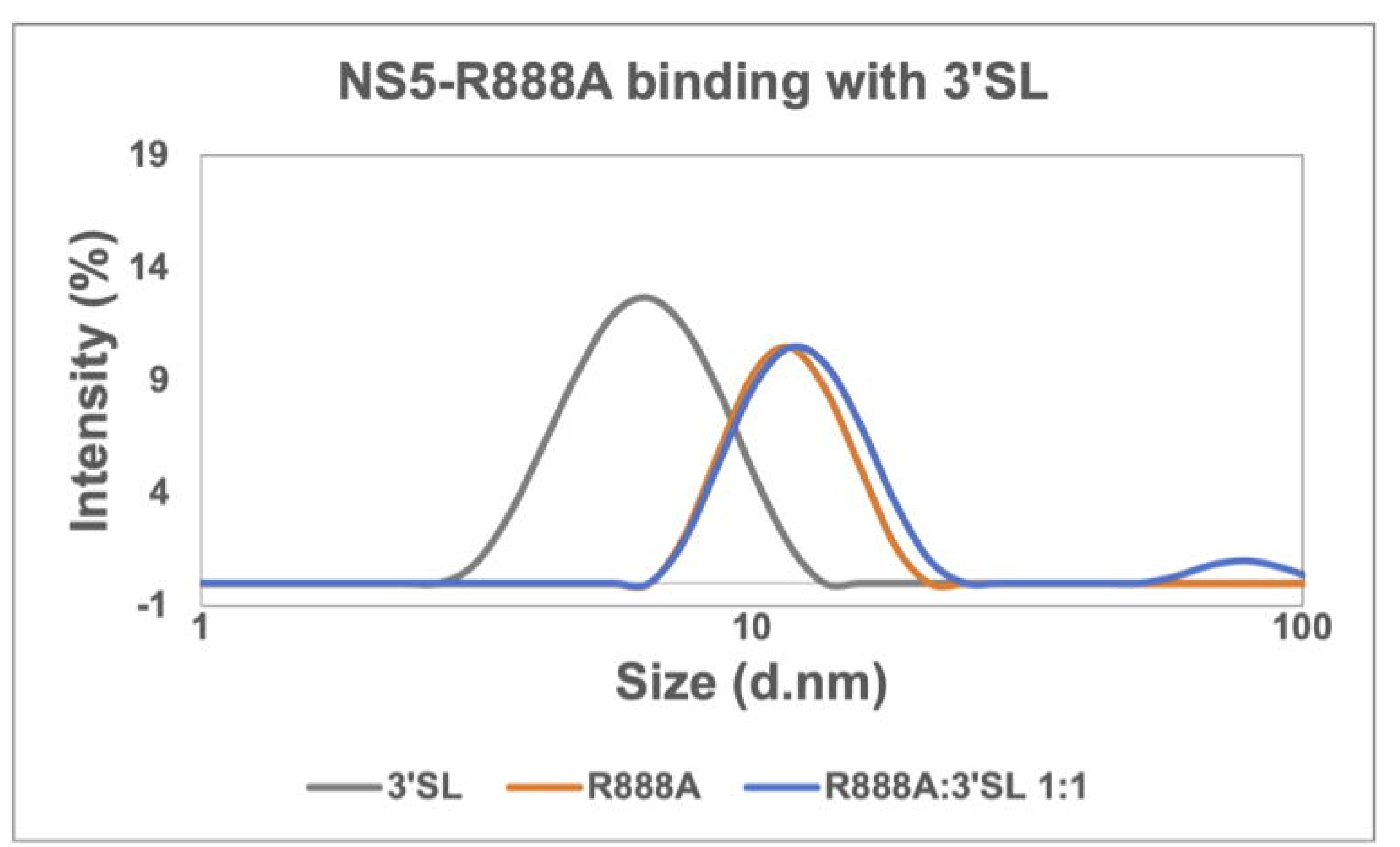




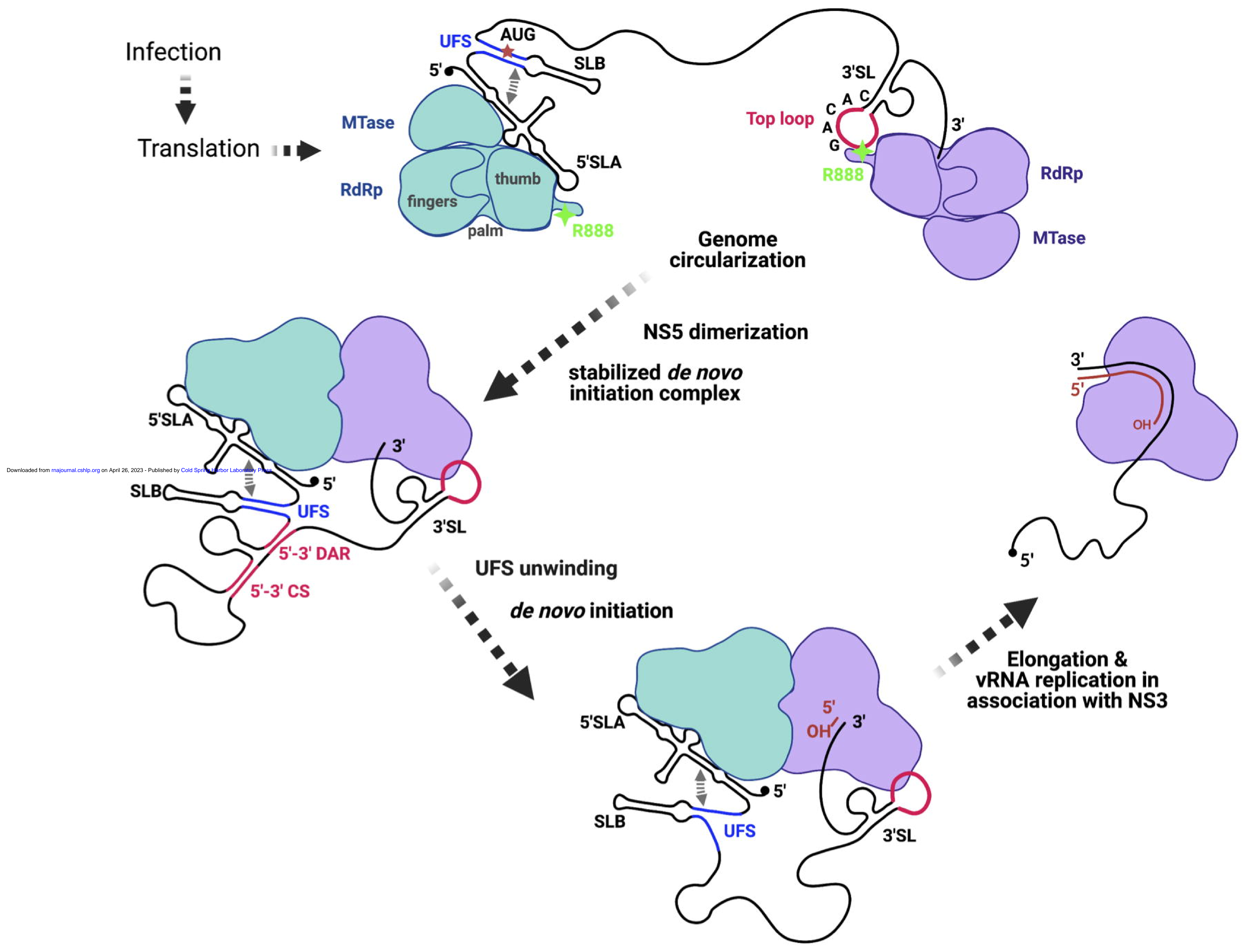



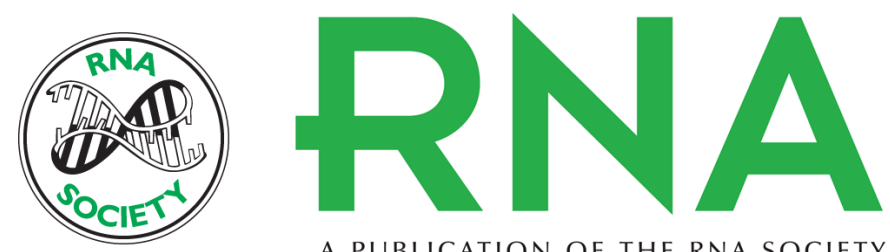

A PUBLICATION OF THE RNA SOCIETY

\section{A conserved arginine in NS5 binds genomic 3'stem-loop RNA for primer-independent initiation of flavivirus RNA-replication}

Sai Wang, Kitt Wing Ki Chan, Min Jie Alvin Tan, et al.

RNA published online November 10, 2021

Supplemental Material

$\mathbf{P}<\mathbf{P} \quad$ Published online November 10, 2021 in advance of the print journal.

Accepted Peer-reviewed and accepted for publication but not copyedited or typeset; accepted Manuscript

Open Access

Creative Commons License

Email Alerting Service

http://rnajournal.cshlp.org/content/suppl/2021/11/10/rna.078949.121.DC1

manuscript is likely to differ from the final, published version.

Freely available online through the RNA Open Access option.

This article, published in $R N A$, is available under a Creative Commons License (Attribution 4.0 International), as described at http://creativecommons.org/licenses/by/4.0/.

Receive free email alerts when new articles cite this article - sign up in the box at the top right corner of the article or click here.

To subscribe to $R N A$ go to:

http://rnajournal.cshlp.org/subscriptions 